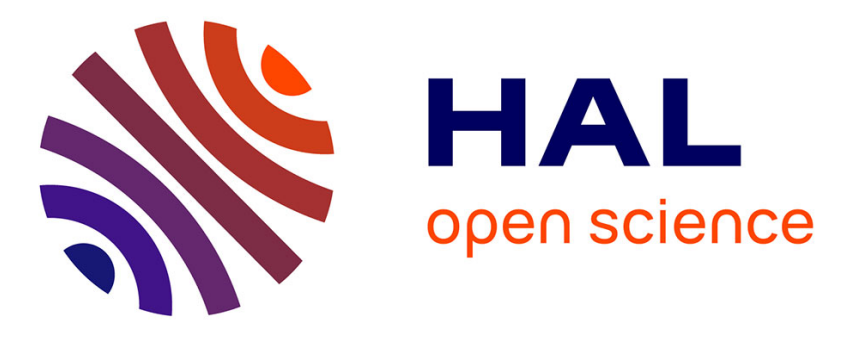

\title{
Controlling charge quantization with quantum fluctuations
}

Sébastien Jezouin, Z. Iftikhar, A. Anthore, F. D. Parmentier, U. Gennser, A. Cavanna, A. Ouerghi, I P Levkivskyi, E. Idrisov, E.V. Sukhorukov, et al.

\section{- To cite this version:}

Sébastien Jezouin, Z. Iftikhar, A. Anthore, F. D. Parmentier, U. Gennser, et al.. Controlling charge quantization with quantum fluctuations. Nature, 2016, 77, pp.191 - 62. 10.1038/nature19072 . cea01486009

\section{HAL Id: cea-01486009 https://hal-cea.archives-ouvertes.fr/cea-01486009}

Submitted on 9 Mar 2017

HAL is a multi-disciplinary open access archive for the deposit and dissemination of scientific research documents, whether they are published or not. The documents may come from teaching and research institutions in France or abroad, or from public or private research centers.
L'archive ouverte pluridisciplinaire $\mathbf{H A L}$, est destinée au dépôt et à la diffusion de documents scientifiques de niveau recherche, publiés ou non, émanant des établissements d'enseignement et de recherche français ou étrangers, des laboratoires publics ou privés. 


\title{
Controlling charge quantization with quantum fluctuations
}

\author{
S. Jezouin ${ }^{1 *}$, Z. Iftikhar ${ }^{1 *}$, A. Anthore ${ }^{1}$, F. D. Parmentier ${ }^{1}$, U. Gennser ${ }^{1}$, A. Cavanna ${ }^{1}$, A. Ouerghi ${ }^{1}$, I. P. Levkivskyi ${ }^{2}$, E. Idrisov $^{3}$, \\ E. V. Sukhorukov ${ }^{3}$, L. I. Glazman ${ }^{4} \&$ F. Pierre ${ }^{1}$
}

In 1909, Millikan showed that the charge of electrically isolated systems is quantized in units of the elementary electron charge $e$. Today, the persistence of charge quantization in small, weakly connected conductors allows for circuits in which single electrons are manipulated, with applications in, for example, metrology, detectors and thermometry ${ }^{1-5}$. However, as the connection strength is increased, the discreteness of charge is progressively reduced by quantum fluctuations. Here we report the full quantum control and characterization of charge quantization. By using semiconductor-based tunable elemental conduction channels to connect a micrometre-scale metallic island to a circuit, we explore the complete evolution of charge quantization while scanning the entire range of connection strengths, from a very weak (tunnel) to a perfect (ballistic) contact. We observe, when approaching the ballistic limit, that charge quantization is destroyed by quantum fluctuations, and scales as the square root of the residual probability for an electron to be reflected across the quantum channel; this scaling also applies beyond the different regimes of connection strength currently accessible to theor ${ }^{6-8}$. At increased temperatures, the thermal fluctuations result in an exponential suppression of charge quantization and in a universal square-root scaling, valid for all connection strengths, in agreement with expectations ${ }^{8}$. Besides being pertinent for the improvement of single-electron circuits and their applications, and for the metal-semiconductor hybrids relevant to topological quantum computing ${ }^{9}$, knowledge of the quantum laws of electricity will be essential for the quantum engineering of future nanoelectronic devices.

Some of the most fundamental theoretical predictions have so far eluded experimental confirmation. Charging effects are generally found to diminish as the conductances of the contacts are increased ${ }^{10-18}$; however, although some measurements support the fundamental prediction $^{6-8}$ that charge quantization vanishes in the presence of one ballistic channel ${ }^{10-12,17}$, others conclude the opposite ${ }^{18-23}$. Unsurprisingly, the scaling behaviour predicted for the reduction of charge quantization ${ }^{6-8}$ has also remained elusive, until now, despite several attempts ${ }^{16,17}$.

A plausible explanation of the varying results regarding the charge quantization criteria is that, in the previously investigated devices, the quantum channels and the conductor were not completely distinct circuit elements. With a small island, in which the density of states is discrete, the non-local electronic wave functions merge the connected channels and the island into a complex quantum conductor, where Coulomb interactions may play a non-trivial role. As a result, charging effects can develop even if one of the conduction channels taken separately is perfectly ballistic. This phenomenon is called mesoscopic Coulomb blockade $\mathrm{e}^{18,22,24}$.

Investigating charge quantization at the most elemental singlechannel level therefore requires tunable conduction channels linked to a conductor with a negligible electronic level spacing. Although this can be realized by making the island larger, its size must remain small enough to preserve charge quantization. Indeed, thermal fluctuations average out charge quantization unless the charging energy associated with the addition of one electron in the island $-E_{\mathrm{C}}=e^{2} / 2 C$, where the geometrical capacitance of the island $C$ increases with size-is larger than the thermal energy $k_{\mathrm{B}} T$, with $k_{\mathrm{B}}$ the Boltzmann constant and $T$ the temperature ${ }^{1,2}$.

We have solved these conflicting requirements with the hybrid metal-semiconductor single-electron transistor (SET) shown in Fig. 1a, implementing the schematic circuit of Fig. 1b: a central metallic island with a continuous density of states (coloured red in Fig. 1a, b) is connected to large electrodes (represented by white disks) through two $\mathrm{Ga}(\mathrm{Al})$ As quantum point contacts $\left(\mathrm{QPC}_{\mathrm{L}, \mathrm{R}}\right)$ that emulate single-channel quantum conductors over the entire range of coupling strengths.

The metallic island, which is made of a metallic AuGeNi alloy, has a negligible electronic level spacing $\delta \approx k_{\mathrm{B}} \times 0.2 \mu \mathrm{K}$, five orders of magnitude smaller than the base electronic temperature $T \approx 17 \mathrm{mK}$. It is galvanically connected, by thermal annealing, to a $105-\mathrm{nm}$-deep, $\mathrm{Ga}(\mathrm{Al})$ As, high-mobility two-dimensional electron gas (2DEG; darker grey areas delimited by bright lines in Fig. 1a). Achieving an almost perfectly transparent metal-2DEG electrical contact is crucial to reach the ballistic channel limit. Remarkably, the reflection probability of electrons at the interface is below $0.05 \%$.

The QPCs are located in the 2DEG and tuned by field effect with the voltage applied to capacitively coupled metallic split gates (coloured green in Fig. 1a; the top-right split gates that are coloured yellow are negatively biased to remove the $2 \mathrm{DEG}$ underneath). Besides tuning, the precise characterization of each QPC, independently, is necessary for the quantitative exploration of charge quantization versus connection strength. However, in the SET configuration, the QPC conductances are interconnected and renormalized by Coulomb blockade. Moreover, only their series combination is accessible. To completely characterize $\mathrm{QPC}_{\mathrm{L}, \mathrm{R}}$, we implemented with adjacent gates (coloured blue in Fig. 1a) the on-chip switches shown in Fig. 1b. The measured quantities $\tau_{\mathrm{L}, \mathrm{R}} \equiv G_{\mathrm{L}, \mathrm{R}}^{\mathrm{qp}} h / e^{2}$ (with $h$ the Planck constant and $G_{\mathrm{L}, \mathrm{R}}^{\mathrm{qpc}}$ the conductances of $\mathrm{QPC}_{\mathrm{L}, \mathrm{R}}$ when switches are closed (inset of Fig. 1c)) directly give the 'intrinsic' (not renormalized by Coulomb blockade) electron transmission probabilities of the constitutive quantum channels, which fully characterize the connection strength to the metallic island. As illustrated in Fig. $1 c, \tau_{\mathrm{L}(\mathrm{R})} \leq 1$ corresponds to a single (spin-polarized, see below) channel of transmission probability $\tau_{\mathrm{L}(\mathrm{R})}$ across $\mathrm{QPC}_{\mathrm{L}(\mathrm{R})}$. For $1<\tau_{\mathrm{R}} \leq 2$, there are two channels across $\mathrm{QPC}_{\mathrm{R}}$ - one fully ballistic and the other with transmission probability $\tau_{\mathrm{R}}-1$. With this approach, we achieve an accuracy down to $0.1 \%$ near the ballistic limit.

The sample is immersed into a perpendicular magnetic field $B \approx 4 \mathrm{~T}$, which corresponds to the integer quantum Hall effect at filling

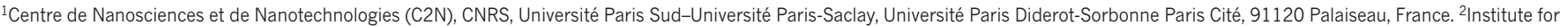

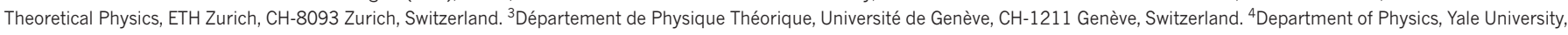
New Haven, Connecticut 06520, USA.

*These authors contributed equally to this work. 


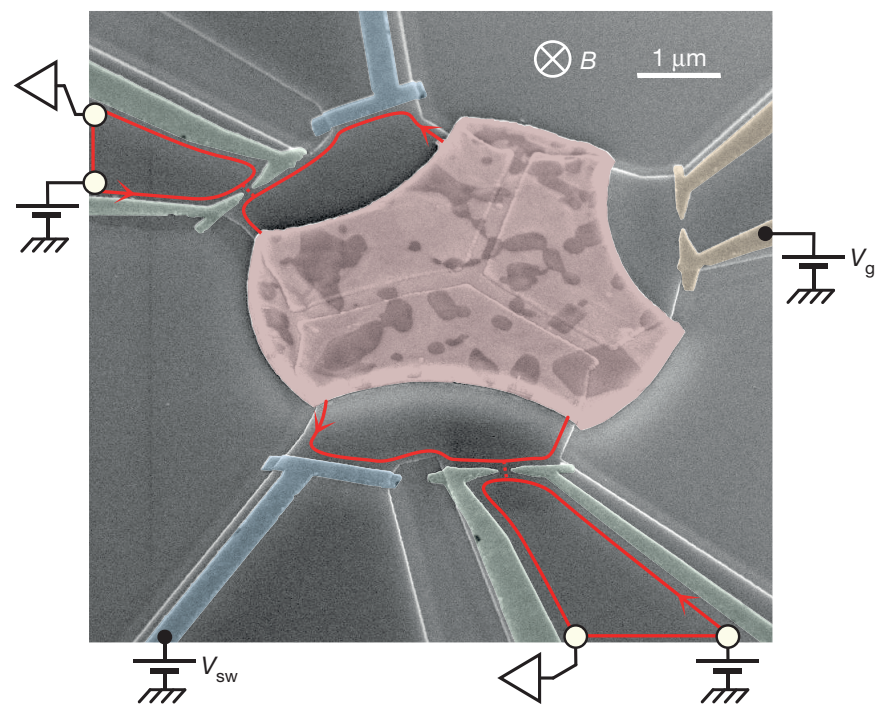

b

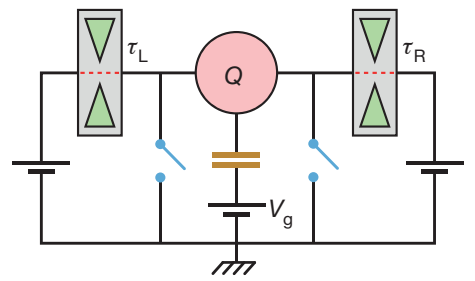

d



Figure 1 | Tunable quantum connection to a metallic island. a, Coloured sample micrograph. A micrometre-scale metallic island (red) is connected to large electrodes (white circles) through two quantum point contacts (QPCs, green split gates) formed in a buried two-dimensional electron gas (2DEG; darker grey delimited by bright lines). The lateral gates (blue) implement short-circuit switches as shown in $\mathbf{b}$. The yellow gates, tuned at $V_{\mathrm{g}}$ negative enough to deplete the 2DEG underneath, are capacitively coupled to the island and used to evidence charge quantization. In the applied field $B \approx 4 \mathrm{~T}$, the current propagates along two edge channels (red lines) in the direction indicated by arrows. b, Sample schematic; colours as in $\mathbf{a}$; $Q$ represents the excess charge that can accumulate on the

factor $\nu=2$. In this regime, the electrical current propagates along two edge channels (shown as a single red line in Fig. 1a) in the direction indicated by arrows, which does not influence charge quantization (for a specific discussion see Methods section 'Conductance in the near-ballistic regime with strong thermal fluctuations'). The large Zeeman splitting results in the full separation between the successive openings of the two spin-polarized quantum channels across the QPCs (Fig. 1c).

Charge quantization in the central island is unequivocally evidenced by periodic oscillations of the SET differential conductance $G_{\mathrm{SET}}$ (across $\mathrm{QPC}_{\mathrm{L}}$-island- $\mathrm{QPC}_{\mathrm{R}}$ ) when sweeping a capacitively coupled gate voltage, which develop into Coulomb diamonds with d.c. bias voltage $V_{\mathrm{dc}}$ (Fig. 1d). With both QPCs in the tunnel regime, $\tau_{\mathrm{L}, \mathrm{R}} \ll 1$, the span of the diamonds in $V_{\mathrm{dc}}$ gives the charging energy $E_{\mathrm{C}} \approx k_{\mathrm{B}} \times 0.3 \mathrm{~K}$ $(C \approx 3.1 \mathrm{fF})$.

We first probe the evolution of charge quantization with transmission probability directly from $G_{\mathrm{SET}}$ raw periodic modulations. Figure 2a

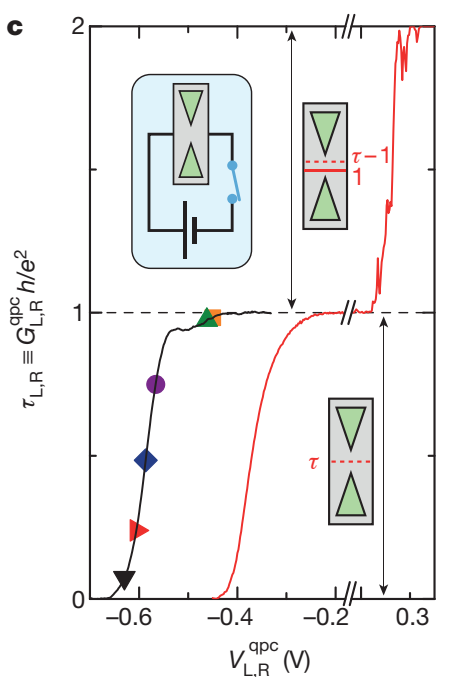

metallic island. c, The 'intrinsic' (switch closed; see inset schematic) conductance $G_{\mathrm{L}, \mathrm{R}}^{\mathrm{qpc}}$ across $\mathrm{QPC}_{\mathrm{L}, \mathrm{R}}$ (shown top-right and bottom left, respectively, in a) is shown versus split gate voltage $V_{\mathrm{L}, \mathrm{R}}^{\mathrm{qp}}$ as black $(\mathrm{L})$ and red (R) lines. Symbols indicate the set-points of $\mathrm{QPC}_{\mathrm{L}}$ used thereafter. The number and transmission probabilities of electronic channels through the QPC (pair of green triangles) are schematized for $\tau_{\mathrm{R}}<1$ and $\tau_{\mathrm{R}}>1$ : a dashed (solid) red line represents a partially (perfectly) transmitted channel. d, Coulomb diamond patterns in the device conductance $G_{\text {SET }}$ (larger shown brighter, from 0 in dark blue up to $0.13 e^{2} / h$ in white) measured versus gate $\left(V_{\mathrm{sw}}\right)$ and bias $\left(V_{\mathrm{dc}}\right)$ voltages for tunnel contacts $\tau_{\mathrm{L}, \mathrm{R}} \ll 1$.

displays $G_{\text {SET }}$ measured at $T \approx 17 \mathrm{mK}$ and $V_{\mathrm{dc}}=0$ while sweeping the capacitively coupled gate voltage $V_{\mathrm{g}}$ (Fig. 1a), for $\mathrm{QPC}_{\mathrm{L}}$ fixed to $\tau_{\mathrm{L}}=0.24$ and with each panel corresponding to a different $\mathrm{QPC}_{\mathrm{R}}$ tuning $\left(\tau_{\mathrm{R}}=0.1,0.6,0.88,0.98\right.$ and 1.5 , from left to right). These raw data reveal the remarkable robustness of charge quantization to connection strength. At $\tau_{\mathrm{R}}=0.1$ and $\tau_{\mathrm{R}}=0.6$, the presence of sharp periodic peaks separated by intervals in which $G_{\text {SET }} \approx 0$ signals an essentially unaltered charge quantization over the greater part of transmission probabilities. Although $G_{\mathrm{SET}}\left(\delta V_{\mathrm{g}}\right)$ progressively evolves with increasing $\tau_{\mathrm{R}}<1$ into a sinusoid with non-zero minima, relatively important modulations of fixed ( $\tau_{\mathrm{R}}$-independent $)$ period persist very close to the ballistic limit, at $\tau_{\mathrm{R}}=0.98$. In stark contrast, $G_{\mathrm{SET}}$ is independent of $V_{\mathrm{g}}$ at $\tau_{\mathrm{R}}=1.5$, confirming the predicted complete collapse of charge quantization in the presence of a fully ballistic channel. Note that $G_{\text {SET }}$ remains reduced by Coulomb interactions, even at $\tau_{R}=1.5$, as evidenced by the pronounced conductance dip at low $V_{\mathrm{dc}}$ (inset of Fig. 2b). 

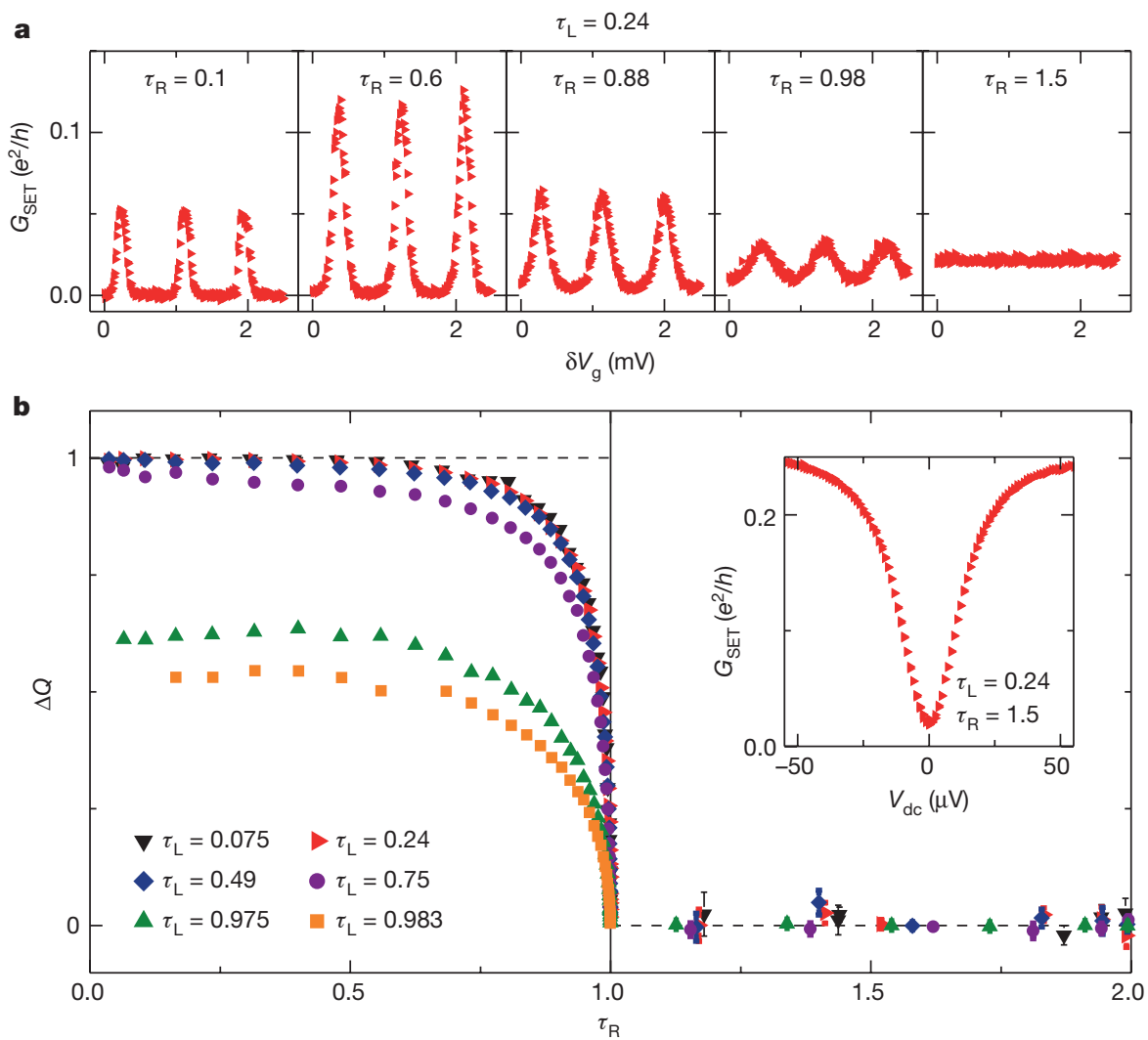

Figure 2 | Charge quantization versus connection strength at $T \approx \mathbf{1 7} \mathbf{m K}$. a, Conductance sweeps $G_{\mathrm{SET}}\left(\delta V_{\mathrm{g}}\right)$ with fixed $\tau_{\mathrm{L}}=0.24$ and varying $\tau_{\mathrm{R}}=0.1,0.6,0.88,0.98$ and 1.5 , from left to right, as indicated. b, Visibility of $G_{\mathrm{SET}}$ oscillations $\Delta Q \equiv\left(G_{\mathrm{SET}}^{\max }-G_{\mathrm{SET}}^{\min }\right) /\left(G_{\mathrm{SET}}^{\max }+G_{\mathrm{SET}}^{\min }\right)$ versus $\tau_{\mathrm{R}}$, with each set of symbols corresponding to a different $\mathrm{QPC}_{\mathrm{L}}$ set-point $\tau_{\mathrm{L}}$, as indicated, corresponding to those indicated by the matching symbols in Fig. 1c. Inset, dynamical Coulomb blockade renormalization of $G_{S E T}$ versus d.c. voltage $V_{\mathrm{dc}}$ in the absence of charge quantization, at $\tau_{\mathrm{L}}=0.24$ and $\tau_{R}=1.5$. The error bars are the standard error on the mean value of $\Delta Q$, obtained from the statistical uncertainty of about ten measurements of $G_{\text {SET }}$ (see Methods).

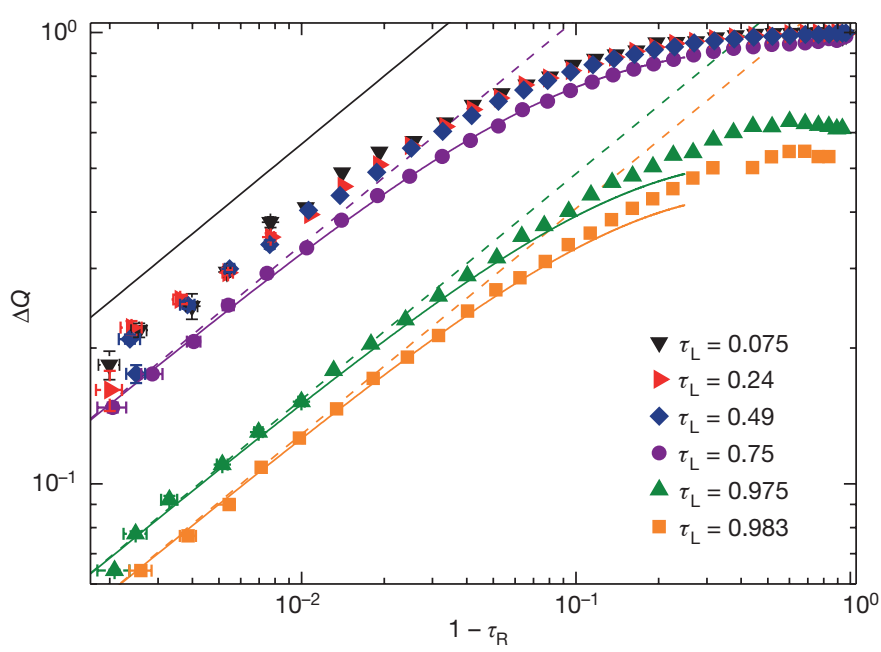

Figure 3 | Charge quantization scaling near the ballistic critical point. The $\Delta Q$ data at $T \approx 17 \mathrm{mK}$ are displayed versus $1-\tau_{\mathrm{R}}$ on a log-log scale, with different symbols for the different $\mathrm{QPC}_{\mathrm{L}}$ set-points, as in Fig. 2. Solid lines are quantitative predictions (no fit parameters) derived assuming $k_{\mathrm{B}} T \ll E_{\mathrm{C}}, 1-\tau_{\mathrm{R}} \ll 1$ and either $\tau_{\mathrm{L}} \ll 1$ (top (black) line) or $1-\tau_{\mathrm{L}} \ll 1$ (bottom three (purple, green and orange) lines). The power law $\Delta Q \propto \sqrt{1-\tau_{\mathrm{R}}}$ (straight, dashed lines) is systematically observed for $1-\tau_{\mathrm{R}} \lesssim 0.02$ and at intermediate $\tau_{\mathrm{L}}$. The horizontal error bars arise from the dispersion of at least 40 transmission settings; the vertical error bars are calculated from the statistical uncertainty of about 10 measurements of one period of $G_{\text {SET }}$ (see Methods).
Indeed, the so-called dynamical Coulomb blockade does not rely on a quantized island charge, but results from the discreteness of charge transfers across non-ballistic channels ${ }^{1,2}$.

The degree of charge quantization versus connection strength is characterized, separately from the dynamical Coulomb blockade renormalization of the channels, by focusing on the visibility of the periodic modulations $\Delta Q \equiv\left(G_{\mathrm{SET}}^{\max }-G_{\mathrm{SET}}^{\min }\right) /\left(G_{\mathrm{SET}}^{\max }+G_{\mathrm{SET}}^{\min }\right)$, with $G_{\mathrm{SET}}^{\max (\min )}$ the maximum (minimum) SET conductance over one gate-voltage period and, from now on, $V_{\mathrm{dc}}=0$. A visibility $\Delta Q=1(\Delta Q=0)$ signals a full (an absence of) charge quantization. Moreover, the visibility $\Delta Q$ is directly proportional to the charge oscillations of the island with gate voltage (that is, charge quantization) when one channel approaches the ballistic limit (for example, $\left.\tau_{\mathrm{R}} \rightarrow 1\right)^{7,25-27}$. As put forward in ref. 26, this proportionality coefficient reduces to the numerical factor $e /(2 \pi \times 1.59)$ for $\tau_{\mathrm{L}} \ll 1$ and $k_{\mathrm{B}} T \ll E_{\mathrm{C}}$.

Figure $2 \mathrm{~b}$ shows $\Delta Q$ versus $\tau_{\mathrm{R}}$ at $T \approx 17 \mathrm{mK}$, with each set of symbols corresponding to a different tuning of the second QPC $\left(\tau_{\mathrm{L}} \in\{0.075\right.$, $0.24,0.49,0.75,0.975,0.983\})$. The robustness of charge quantization with the connection strength of one channel $\left(\tau_{R}\right)$ is established independently of the second channel $\left(\tau_{L}\right)$, from the nearly constant $\Delta Q$ for $\tau_{\mathrm{R}} \lesssim 0.6$. When further increasing $\tau_{\mathrm{R}}, \Delta Q$ noticeably diminishes and systematically collapses to zero precisely at the ballistic critical point $\tau_{\mathrm{R}}=1$. For $\tau_{\mathrm{R}} \geq 1$, in the presence of one ballistic channel, $\Delta Q$ remains perfectly null at experimental accuracy (see Methods for additional tests).

Power laws characterizing the scaling of charge quantization as $\tau_{\mathrm{R}} \rightarrow 1$ are best revealed by plotting $\Delta Q$ versus the 'distance' from the ballistic critical point $1-\tau_{\mathrm{R}}>0$ on a log-log scale. As shown in Fig. 3, the $T=17 \mathrm{mK}$ data (symbols) systematically vanish as $\sqrt{1-\tau_{\mathrm{R}}}$ (straight lines) for $1-\tau_{\mathrm{R}} \lesssim 0.02$. 

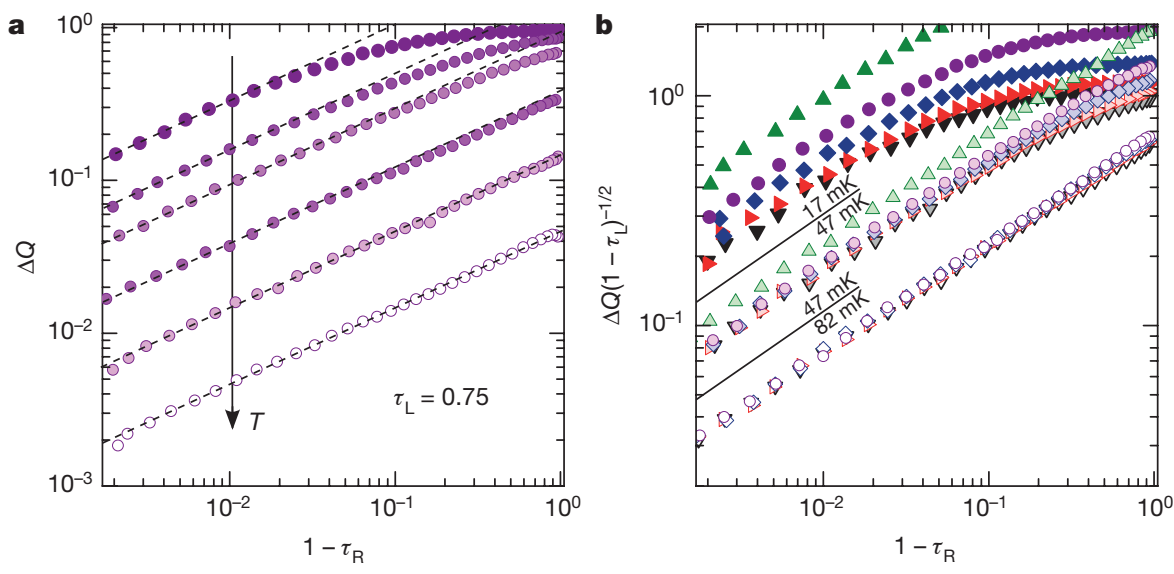



Figure 4 | Crossover to a universal charge quantization scaling as temperature is increased. a, Symbols display $\Delta Q$ versus $1-\tau_{\mathrm{R}}$ at $\tau_{\mathrm{L}}=0.75$ and for $T \approx 17 \mathrm{mK}, 32 \mathrm{mK}, 47 \mathrm{mK}, 82 \mathrm{mK}, 119 \mathrm{mK}$ and $166 \mathrm{mK}$, from top to bottom. The $\tau_{\mathrm{R}}$ range over which $\Delta Q \propto \sqrt{1-\tau_{\mathrm{R}}}$ (straight, dashed lines) extends up to the full interval $\tau_{\mathrm{R}} \in[0,1]$ when increasing $T$. b. The rescaled $\Delta Q / \sqrt{1-\tau_{\mathrm{L}}}$ is shown versus $1-\tau_{\mathrm{R}}$, with a different set of symbols corresponding to different $\mathrm{QPC}_{\mathrm{L}}$ set-points as in $\mathrm{c}$. Solid lines separate the data at $T \approx 17 \mathrm{mK}$ (top, darker filling), $T \approx 47 \mathrm{mK}$ (middle) and $T \approx 82 \mathrm{mK}$ (bottom, brighter filling). At $T=82 \mathrm{mK}$, all the data collapse onto a single universal curve $\Delta Q \propto \sqrt{\left(1-\tau_{\mathrm{L}}\right)\left(1-\tau_{\mathrm{R}}\right)}$. c, Symbols

The Coulomb blockade theory of electronic transport in the presence of a nearly ballistic channel $\left(1-\tau_{\mathrm{R}} \ll 1\right)$ relies on the bosonization approach that was initially developed to address correlated electrons in one dimension. Quantitative predictions were obtained for $k_{\mathrm{B}} T \ll E_{\mathrm{C}}$ and for a second channel in either the tunnel $\left(\tau_{\mathrm{L}} \ll 1\right)$ or almost-ballistic $\left(1-\tau_{\mathrm{L}} \ll 1\right)$ regime ${ }^{25,28}$. In both cases, $\Delta Q$ is expected to vanish as $\sqrt{1-\tau_{\mathrm{R}}}$ :

$$
\begin{gathered}
\Delta Q\left(1-\tau_{\mathrm{R}} \ll 1 ; \tau_{\mathrm{L}} \ll 1, k_{\mathrm{B}} T \ll E_{\mathrm{C}}\right) \approx 5.7 \sqrt{1-\tau_{\mathrm{R}}} \\
\Delta Q\left(\sqrt{1-\tau_{\mathrm{L}, \mathrm{R}}} \ll \frac{k_{\mathrm{B}} T}{E_{\mathrm{C}}} \ll 1\right) \approx \frac{0.57 E_{\mathrm{C}}}{k_{\mathrm{B}} T} \sqrt{\left(1-\tau_{\mathrm{L}}\right)\left(1-\tau_{\mathrm{R}}\right)}
\end{gathered}
$$

Such a scaling, initially proposed in ref. 6, was also predicted for the gate-voltage modulation of thermodynamic quantities for multi-channel junctions using an extension ${ }^{8}$ of the instanton technique ${ }^{1,29}$.

The data establishes the $\sqrt{1-\tau_{\mathrm{R}}}$ scaling for arbitrary $\tau_{\mathrm{L}} \in[0,1]$, beyond the tunnel and ballistic limits currently accessible to transport theory. The dashed lines in Fig. 3 display the asymptotic $\left(\sqrt{1-\tau_{\mathrm{L}, \mathrm{R}}} \ll k_{\mathrm{B}} T / E_{\mathrm{C}}\right)$, quantitative predictions of equation (2) for our completely characterized device at $T=17 \mathrm{mK}$, without fitting parameters. The non-asymptotic $\Delta Q$ predictions (equation (1) for $\tau_{\mathrm{L}} \ll 1$; see Methods for $\left.1-\tau_{\mathrm{L}} \ll 1\right)$ are shown versus $1-\tau_{\mathrm{R}}<0.25$ as solid lines. Data and quantitative predictions are indistinguishable for $1-\tau_{\mathrm{R}} \lesssim 0.1$ for $\tau_{\mathrm{L}}=0.983, \tau_{\mathrm{L}}=0.975$ and, more surprisingly, $\tau_{\mathrm{L}}=0.75$. The equation (1) prediction (black line in Fig. 3 ) remains noticeably (about $25 \%$ ) above the $\tau_{\mathrm{L}}=0.075$ data for $1-\tau_{\mathrm{R}} \ll 1$. This numerical difference could result from the finite experimental $T$, because equation (1) is exact only at $T=0$.

We now investigate the ways in which the combination of thermal and quantum fluctuations impacts the quantization of charge. As temperature rises, the population of additional charge states is expected to average out charge quantization ${ }^{1,2}$. Figure 4 a displays the measured $\Delta Q$ (symbols) versus $1-\tau_{\mathrm{R}}$ at different temperatures, from $T=17 \mathrm{mK}$ (darker filling) to $T=166 \mathrm{mK}$ (brighter filling), for the representative $\mathrm{QPC}_{\mathrm{L}}$ setting $\tau_{\mathrm{L}}=0.75$. As naively expected, $\Delta Q$ decreases as $T$ increases. In line with thermodynamic expectations ${ }^{8}$ (Methods), the $\Delta Q \propto \sqrt{1-\tau_{\mathrm{R}}}$ scaling (straight lines) that originates from quantum display the fully rescaled data $\Delta Q / \sqrt{\left(1-\tau_{\mathrm{L}}\right)\left(1-\tau_{\mathrm{R}}\right)}$ versus $T$ on semi-log scale, extracted in the regime in which $1-\tau_{\mathrm{R}}$ is small enough that $\Delta Q \propto \sqrt{1-\tau_{\mathrm{R}}}$; data for $\tau_{\mathrm{L}}=0.975$ are plotted only for $T \leq 47 \mathrm{mK}$. Horizontal error bars represent the experimental temperature uncertainty at $T=17 \pm 4 \mathrm{mK}$ and $T=32 \pm 1 \mathrm{mK}$. Solid lines are the quantitative predictions in the quantum regime $k_{\mathrm{B}} T \ll E_{\mathrm{C}}$, given by equation (1) (black, horizontal) and equation (2) (green, curved). The straight dashed line displays an exponential decay close to predictions in the presence of strong thermal fluctuations (see text).

fluctuations not only persists for increasing $T$, but extends over a widening range of $\tau_{\mathrm{R}}$, up to the full-scale $\tau_{\mathrm{R}} \in[0,1]$.

The crossover towards this universal behaviour is established by comparing the rescaled visibility $\Delta Q / \sqrt{1-\tau_{\mathrm{L}}}$ for different $\tau_{\mathrm{L}}$ settings with $1-\tau_{\mathrm{R}}$. The symbols in Fig. $4 \mathrm{~b}$ represent the rescaled data at $T=17 \mathrm{mK}, T=47 \mathrm{mK}$ and $T=82 \mathrm{mK}$, with brighter filling at higher temperatures. As $T$ increases, the scatter associated with the various $\tau_{\mathrm{L}}$ values narrows. For $T \geq 82 \mathrm{mK}$, the rescaled data collapse onto a single, universal (for all $\tau_{\mathrm{L}}$ ), straight line $\Delta Q \propto \sqrt{\left(1-\tau_{\mathrm{L}}\right)\left(1-\tau_{\mathrm{R}}\right)}$ over the full range $\tau_{\mathrm{L}, \mathrm{R}} \in[0,1]$.

The temperature dependence is further characterized by plotting $\Delta Q / \sqrt{\left(1-\tau_{\mathrm{L}}\right)\left(1-\tau_{\mathrm{R}}\right)}$ (determined at low enough $1-\tau_{\mathrm{R}}$ such that $\Delta Q \propto \sqrt{1-\tau_{\mathrm{R}}}$ ) versus temperature on a semi-log scale (Fig. $4 \mathrm{c}$, symbols). The $k_{\mathrm{B}} T \ll E_{\mathrm{C}}$ prediction of equation (1) (equation (2)) is displayed as a black (green) solid line for $T<75 \mathrm{mK}(T<115 \mathrm{mK})$. We find for $T \geq 82 \mathrm{mK}$ (up to $166 \mathrm{mK}, 2.8 \leq \pi^{2} k_{\mathrm{B}} T / E_{\mathrm{C}} \leq 5.6$ ) that the different $\tau_{\mathrm{L}}$ data points collapse onto the same exponential decay (dashed line in Fig. $4 \mathrm{c}): \Delta Q \approx \sqrt{\left(1-\tau_{\mathrm{L}}\right)\left(1-\tau_{\mathrm{R}}\right)} \exp \left(-0.8 \pi^{2} k_{\mathrm{B}} T / E_{\mathrm{C}}\right)$. We have extended the Coulomb blockade theory for the conductance to include thermal fluctuations in the limits of tunnel or nearly ballistic channels (Methods). In the regime of strong thermal averaging, we predict $\Delta Q \propto \sqrt{\left(1-\tau_{\mathrm{L}}\right)\left(1-\tau_{\mathrm{R}}\right)} \exp \left(-\pi^{2} k_{\mathrm{B}} T / E_{\mathrm{C}}\right.$ ) (neglecting factors not exponential in $T$ ) - a dependence that is also expected for thermodynamic properties ${ }^{8}$ (Methods) - in close agreement with the experimental findings regarding the effect of $\tau_{\mathrm{L}, \mathrm{R}}$ and $T$.

Although theoretical predictions for low-temperature transport currently apply to only the nearly ballistic and tunnel limits, we anticipate that recent advances, including those in numerical renormalization group methods ${ }^{30}$, will open up access to the full range of connection strengths. Our results may therefore provide a test-bed for strongly correlated electron-theoretical methods, for which non-perturbative techniques are ubiquitous. The understanding and on-demand control of charge quantization in mesoscopic circuits might lead to applications beyond the field of single electronics. The central role of charge quantization in the different quantum laws of electricity with coherent conductors indicates that direct quantum engineering could have implications for future nanoelectronics, such as semiconductor-metal hybrid devices that are crucial for developing topologically protected quantum bits 9 . The hybrid implementation we have presented also enables further 
fundamental exploration, including of charge quantization with correlated electrons such as in the multi-channel Kondo regime and/or with fractionally charged anyonic quasiparticles.

Online Content Methods, along with any additional Extended Data display items and Source Data, are available in the online version of the paper; references unique to these sections appear only in the online paper.

\section{Received 25 March; accepted 10 June 2016.}

1. Schön, G. \& Zaikin, A. D. Quantum coherent effects, phase transitions, and the dissipative dynamics of ultra small tunnel junctions. Phys. Rep. 198, 237-412 (1990).

2. Grabert, H. \& Devoret, M. H. (eds). Single Charge Tunneling: Coulomb Blockade Phenomena in Nanostructures (Plenum, 1992).

3. Likharev, K. K. Single-electron devices and their applications. Proc. IEEE 87, 606-632 (1999).

4. Meschke, M., Engert, J., Heyer, D. \& Pekola, J. P. Comparison of Coulomb blockade thermometers with the International Temperature Scale PLTS-2000. Int. J. Thermophys. 32, 1378-1386 (2011).

5. Pekola, J. P. et al. Single-electron current sources: toward a refined definition of the ampere. Rev. Mod. Phys. 85, 1421-1472 (2013)

6. Flensberg, K. Capacitance and conductance of mesoscopic systems connected by quantum point contacts. Phys. Rev. B 48, 11156-11166 (1993)

7. Matveev, K. A. Coulomb blockade at almost perfect transmission. Phys. Rev. B 51, 1743-1751 (1995)

8. Nazarov, Y. V. Coulomb blockade without tunnel junctions. Phys. Rev. Lett. 82, 1245-1248 (1999)

9. Albrecht, S. M. et al. Exponential protection of zero modes in Majorana islands. Nature 531, 206-209 (2016).

10. Kouwenhoven, L. et al. Single electron charging effects in semiconductor quantum dots. Z. Phys. B 85, 367-373 (1991).

11. Staring, A. A. M., Williamson, J. G., van Houten, H. \& Beenakker, C. W. J. Coulomb-blockade oscillations in a quantum dot. Physica B 175, 226-230 (1991).

12. van der Vaart, N. et al. Charging effects in quantum dots at high magnetic fields. Physica B 189, 99-110 (1993).

13. Molenkamp, L. W., Flensberg, K. \& Kemerink, M. Scaling of the Coulomb energy due to quantum fluctuations in the charge on a quantum dot. Phys. Rev. Lett. 75, 4282-4285 (1995).

14. Joyez, P., Bouchiat, V., Esteve, D., Urbina, C. \& Devoret, M. H. Strong tunneling in the single-electron transistor. Phys. Rev. Lett. 79, 1349-1352 (1997)

15. Chouvaev, D., Kuzmin, L. S., Golubev, D. S. \& Zaikin, A. D. Strong tunneling and Coulomb blockade in a single-electron transistor. Phys. Rev. B 59, 10599-10602 (1999).

16. Berman, D., Zhitenev, N. B., Ashoori, R. C. \& Shayegan, M. Observation of quantum fluctuations of charge on a quantum dot. Phys. Rev. Lett. 82, 161-164 (1999).

17. Duncan, D. S., Livermore, C., Westervelt, R. M., Maranowski, K. D. \& Gossard, A. C. Direct measurement of the destruction of charge quantization in a single-electron box. Appl. Phys. Lett. 74, 1045-1047 (1999).
18. Amasha, S. et al. Coulomb blockade in an open quantum dot. Phys. Rev. Lett. 107, 216804 (2011).

19. Pasquier, C. et al. Quantum limitation on Coulomb blockade observed in a 2D electron system. Phys. Rev. Lett. 70, 69-72 (1993).

20. Crouch, C. H., Livermore, C., Westervelt, R. M., Campman, K. L. \& Gossard, A. C. Coulomb oscillations in partially open quantum dots. Superlattices Microstruct. 20, 377-381 (1996)

21. Liang, C.-T. et al. Experimental evidence for Coulomb charging effects in an open quantum dot at zero magnetic field. Phys. Rev. Lett. 81, 3507-3510 (1998).

22. Cronenwett, S. M. et al. Mesoscopic Coulomb blockade in one-channel quantum dots. Phys. Rev. Lett. 81, 5904-5907 (1998).

23. Tkachenko, O. A. et al. Coulomb charging effects in an open quantum dot device. J. Phys. Condens. Matter 13, 9515-9534 (2001).

24. Aleiner, I. L. \& Glazman, L. I. Mesoscopic charge quantization. Phys. Rev. B 57, 9608-9641 (1998)

25. Furusaki, A. \& Matveev, K. A. Theory of strong inelastic cotunneling. Phys. Rev. B 52, 16676-16695 (1995).

26. Yi, H. \& Kane, C. L. Coulomb blockade in a quantum dot coupled strongly to a lead. Phys. Rev. B 53, 12956-12966 (1996).

27. Le Hur, K. \& Seelig, G. Capacitance of a quantum dot from the channelanisotropic two-channel Kondo model. Phys. Rev. B 65, 165338 (2002).

28. Matveev, K. A. \& Andreev, A. V. Thermopower of a single-electron transistor in the regime of strong inelastic cotunneling. Phys. Rev. B 66, 045301 (2002).

29. Korshunov, S. E. Coherent and incoherent tunneling in a Josephson junction with a "periodic" dissipation. JETP Lett. 45, 434-436 (1987).

30. Mitchell, A. K., Landau, L. A., Fritz, L. \& Sela, E. Universality and scaling in a charge two-channel Kondo device. Phys. Rev. Lett. 116, 157202 (2016)

Acknowledgements This work was supported by the European Research Council (ERC-2010-StG-20091028, no. 259033), the French RENATECH network, the national French programme 'Investissements d'Avenir' (Labex NanoSaclay, ANR-10-LABX-0035), the US Department of Energy (DE-FG02-08ER46482) and the Swiss National Science Foundation.

Author Contributions S.J. and Z.I. performed the experiment with inputs from A.A. and F.P.; S.J., Z.I., A.A. and F.P. analysed the data; F.D.P. fabricated the sample and contributed to a preliminary experiment; U.G., A.C. and A.O. grew the 2DEG; I.P.L., E.I., E.V.S. and L.I.G. developed the strong thermal fluctuations theory; F.P. led the project and wrote the manuscript with inputs from all authors.

Author Information Reprints and permissions information is available at www.nature.com/reprints. The authors declare no competing financial interests. Readers are welcome to comment on the online version of the paper. Correspondence and requests for materials should be addressed to F.P. (frederic.pierre@lpn.cnrs.fr).

Reviewer Information Nature thanks Y. Nazarov and the other anonymous reviewer(s) for their contribution to the peer review of this work. 


\section{METHODS}

Sample. The sample is nanostructured by standard e-beam lithography in a GaAs/ $\mathrm{Ga}(\mathrm{Al}) \mathrm{As}$ 2DEG located $105 \mathrm{~nm}$ below the surface, of density $2.5 \times 10^{11} \mathrm{~cm}^{-2}$ and mobility $10^{6} \mathrm{~cm}^{2} \mathrm{~V}^{-1} \mathrm{~s}^{-1}$. The ohmic contact between the micrometre-scale metallic island and the buried 2DEG is obtained by thermal diffusion into the semiconductor of a metallic multilayer of nickel $(30 \mathrm{~nm})$, gold $(120 \mathrm{~nm})$ and germanium $(60 \mathrm{~nm})$; see, for example, ref. 31. See methods in ref. 32 for the estimation of the typical energy spacing between electronic levels in the central metallic island on the same sample.

Experimental set-up. The measurements were performed in a dilution refrigerator including multiple filters along the electrical lines and two shields at the mixing chamber. Conductance measurements were carried out by standard lock-in techniques at low frequencies, below $100 \mathrm{~Hz}$, taking advantage of the chiral current propagation in the quantum Hall regime (see Extended Data Fig. 1). Noise measurements for the electronic temperature were performed in the megahertz range using a homemade cryogenic amplifier (for details, see the supplementary information of ref. 33).

Electronic temperature. The displayed electronic temperatures correspond to those extracted on-chip using either quantum shot noise primary thermometry ${ }^{34}$ or thermal noise thermometry, with error bars encapsulating also the outcome of Coulomb blockade oscillations primary thermometry (at $T \leq 32 \mathrm{mK}$ ) and/or standard thermometry from $\mathrm{RuO}_{2}$ resistors thermally anchored to the mixing chamber (at $T \geq 32 \mathrm{mK}$ ).

Interface between the metallic island and the 2DEG. A 2DEG-metallic island transmission probability $\tau_{\Omega \text {-out }}>0.9995$ is obtained with the self-calibrated procedure described below. Here, the switches are set in open positions as in Fig. $1 \mathrm{~b}$ (with edge channels following the red lines shown Fig. 1a and Extended Data Fig. 1). First, $\mathrm{QPC}_{\mathrm{L}, \mathrm{R}}$ are set at $\tau_{\mathrm{L}, \mathrm{R}}=1$, in the middle of the very flat and broad intermediate plateau (owing to the robust quantum Hall effect), and we measure the reflected signal $V_{\mathrm{RR}}^{\tau_{\mathrm{LR}}=1}$ (see Extended Data Fig. 1). The average transmission probability $\tau_{\Omega \text {-out }}$ of the first (outer-edge quantum Hall) channel emitted from $\mathrm{QPC}_{\mathrm{L}}$ and $\mathrm{QPC} \mathrm{R}_{\mathrm{R}}$ into the metallic island then reads:

$$
V_{\mathrm{RR}}^{\tau_{\mathrm{LR}}=1}=G_{\mathrm{R}}\left(1-\tau_{\Omega-\text { out }} / 4\right) V_{\mathrm{R}}
$$

with $V_{\mathrm{R}}$ the (a.c.) voltage applied at the input of $\mathrm{QPC}_{\mathrm{R}}$ (see Extended Data Fig. 1) and $G_{\mathrm{R}}$ the gain of amplification chain R. Second, we eliminate calibration uncertainties by measuring the reflected signal $V_{\mathrm{RR}}^{\tau_{\mathrm{L}} \mathrm{R}=0}=G_{\mathrm{R}} V_{\mathrm{R}}$ with $\mathrm{QPC}_{\mathrm{L}, \mathrm{R}}$ depleted $\left(\tau_{\mathrm{L}, \mathrm{R}}=0\right)$. The ratio $V_{\mathrm{RR}}^{\tau_{\mathrm{LR}}=1} / V_{\mathrm{RR}}^{\tau_{\mathrm{L}, \mathrm{R}}=0}$ gives $\tau_{\Omega \text {-out }}$ directly. With this approach, we obtain $\left|1-\tau_{\Omega \text {-out }}\right|<5 \times 10^{-4}\left(\tau_{\Omega \text {-out }} \approx 0.9997 \pm 0.0002\right)$. The same approach including also the second (inner-edge quantum Hall) channel gives $\tau_{\Omega \text {-in }} \approx 0.9976$. Note that it is usual to have better ohmic contacts with the outer quantum Hall channels, which are closest to the sample edges.

Short-circuit switch operation. In practice, closing the short-circuit switches is realized by changing the voltage applied to the adjacent characterization gate (blue in Fig. 1a, see Extended Data Fig. 2a for the conductance versus gate voltage of switch R) from $-0.35 \mathrm{~V}$ (2DEG depleted/switch open) to $0.1 \mathrm{~V}$ (two edge channels perfectly transmitted/switch closed).

Capacitive crosstalk corrections. The transmission probability across each QPC is slightly modified when changing the voltage applied either to its adjacent characterization gate or to the gate tuning the other QPC. Owing to the large, micrometre-scale distances, this modification remains relatively small, particularly near the ballistic critical point $\left(<1 \%\right.$ for $\tau_{\mathrm{L}, \mathrm{R}} \in[0.9,1]$ when changing the adjacent switch from closed to open). Let us first consider the crosstalk from one QPC to the other, which is more straightforward to extract. For this purpose, the characterization gate adjacent to the QPC, for which the crosstalk is to be compensated, is set to its short-circuit/closed position (as in Fig. 1c) such that the gate-voltage change that tunes the other QPC is felt only through the capacitive crosstalk. We find that this crosstalk can be precisely compensated by a relatively small shift (approximately $-1 \%$ ) of the split gate voltage. Regarding the capacitive crosstalk due to the adjacent characterization gate, the difficulty is to isolate this contribution from changes in the Coulomb blockade renormalization of the QPC conductance. To suppress this renormalization, the other QPC is set in the middle of its $\tau_{\mathrm{L}(\mathrm{R})}=1$ plateau and we apply a large d.c. bias voltage compared to the charging energy. Extended Data Fig. 2b displays the differential conductance of $\mathrm{QPC}_{\mathrm{R}}$, measured in the presence of the applied bias $V_{\mathrm{R}}=72 \mu \mathrm{V}_{\mathrm{dc}}$, versus gate voltage $V_{R}^{\mathrm{qpc}}$ for the adjacent switch set to position open (red line) and closed (blue line). The gate voltage shift $\Delta V_{\mathrm{R}}^{\mathrm{ct}}$ that is needed to compensate the crosstalk is determined at low $\mathrm{QPC}$ conductances $G_{\mathrm{R}}^{\mathrm{qp} c} \lesssim 0.1 e^{2} / h$, for which the d.c. voltage drop across the QPC is nearly independent of the switch position. Extended Data Fig. 2c displays as symbols the crosstalk compensation for $\mathrm{QPC}_{\mathrm{R}}$ in response to increasing the adjacent characterization gate voltage from $V_{\mathrm{R}}^{\mathrm{sw}}=-0.5 \mathrm{~V}$. The amplitude of the negative crosstalk compensation is found to increase linearly, with different slopes for different values of the switch conductance $G_{\mathrm{R}}^{\mathrm{sW}}$. Indeed, the capacitive crosstalk depends on the precise paths of the edge channels, which screen the gate potentials. The crosstalk compensations used in the experiment when setting the adjacent switch from open to closed are $\Delta V_{\mathrm{R}}^{\mathrm{qpc}} \approx-6 \mathrm{mV}$ for $\mathrm{QPC}_{\mathrm{R}}$ and $\Delta V_{\mathrm{L}}^{\mathrm{qpc}} \approx-10 \mathrm{mV}$ for $\mathrm{QPC}_{\mathrm{L}}$.

Calibrations. The reflected signal $V_{\mathrm{RR}}$ is normalized by the signal $V_{\mathrm{RR}}^{\tau_{\mathrm{L}, \mathrm{R}}=0}$ measured when setting $\tau_{\mathrm{L}, \mathrm{R}}=0$. The injection voltage and amplifier gain thereby cancel out in the expression of the SET conductance $G_{\text {SET: }}$

$$
G_{\mathrm{SET}}=\frac{2 e^{2}}{h}\left(1-V_{\mathrm{RR}} / V_{\mathrm{RR}}^{\tau_{\mathrm{LR}}=0}\right)
$$

To reduce the noise level, we also extract $G_{\mathrm{SET}}$ from the (redundant) transmitted signal $V_{\mathrm{LR}}$ (see Extended Data Fig. 1):

$$
G_{\mathrm{SET}}=\frac{2 e^{2}}{h}\left(V_{\mathrm{LR}} / V_{\mathrm{RR}}^{\tau_{\mathrm{L}, \mathrm{R}}=0}\right) G_{\mathrm{R}} / G_{\mathrm{L}}
$$

with $G_{\mathrm{R}}\left(G_{\mathrm{L}}\right)$ the gain of amplification chain $\mathrm{R}(\mathrm{L})$. The ratio $G_{\mathrm{R}} / G_{\mathrm{L}}$ is determined by setting $\mathrm{QPC}_{\mathrm{L}, \mathrm{R}}$ at $\tau_{\mathrm{L}, \mathrm{R}}=1$ and measuring both the signals reflected $\left(V_{\mathrm{RR}}^{\tau_{\mathrm{LR}}=1}\right)$ and transmitted $\left(V_{\mathrm{LR}}^{\tau_{\mathrm{LL}}=1}\right)$ :

$$
\frac{G_{\mathrm{R}}}{G_{\mathrm{L}}}=\frac{1-V_{\mathrm{RR}}^{\tau_{\mathrm{LR}}=1} / V_{\mathrm{RR}}^{\tau_{\mathrm{L}, \mathrm{R}}=0}}{V_{\mathrm{LR}}^{\tau_{\mathrm{LR}}=1} / V_{\mathrm{RR}}^{\tau_{\mathrm{L}, \mathrm{R}}=0}} \approx 1.0105
$$

Experimental determination of $\Delta Q$. For $\tau_{\mathrm{R}} \leq 0.99$, the signal-to-noise ratio is always sufficient to accurately extract the values of $G_{\mathrm{SET}}^{\max \text { min }}$ directly from the periodic conductance maximums and minimums, which stand out very strongly from the background noise. The error bars on the visibility $\Delta Q \equiv\left(G_{\mathrm{SET}}^{\max }-G_{\mathrm{SET}}^{\min }\right) /\left(G_{\mathrm{SET}}^{\max }+G_{\mathrm{SET}}^{\min }\right)$ were calculated from the statistical uncertainty on $G_{\mathrm{SET}}^{\max , \min }$, which is typically estimated from ten different sweeps of one period. In this regime $\left(\tau_{R} \leq 0.99\right)$, the calculated error bars are smaller than the size of the symbol and are therefore not shown.

For $\tau_{\mathrm{R}} \in[0.99,0.998]$, although the periodic oscillations can still be clearly distinguished in the raw data (see Extended Data Fig. 3), the above direct procedure would result in uncertainties that can become quite large, especially at base temperature and in the presence of a weakly transmitted second channel $\left(\tau_{\mathrm{L}}=0.075\right)$. To improve our extraction of $\Delta Q$, we take advantage of the observation that the conductance oscillations are sinusoidal for $\tau_{\mathrm{R}} \geq 0.98$ (see Extended Data Fig. 3), as is expected from theory (see equations (3) and (6), and solid lines in Extended Data Fig. 3): the visibility of the conductance oscillations $\Delta Q$ is then extracted from a sinusoidal fit of the conductance sweeps $G_{\mathrm{SET}}\left(V_{\mathrm{g}}\right)$. The displayed error bars are the statistical error on the mean value obtained from the distinct $\Delta Q$ values obtained by separately fitting approximately six different conductance sweeps. The two procedures give the same value of $\Delta Q$ in the intermediate regime $\tau_{R} \in[0.98,0.99]$ for which they both accurately apply.

For $\tau_{\mathrm{R}} \geq 1$, there are no periodic oscillations directly visible in the raw conductance sweeps $G_{\mathrm{SET}}\left(V_{\mathrm{g}}\right)$ (see right panel in Fig. 2a). To put experimental bounds on the basic statement $\Delta Q \approx 0$, we determined the visibility $\Delta Q$ (displayed Fig. $2 \mathrm{~b}$ ) using the following procedure. First, we determine the most probable positions of the conductance maximums and minimums by 'fitting' a conductance sweep (typically extending over ten Coulomb oscillation periods) with a sinusoidal function at the known period of Coulomb oscillations, using its phase as a fitting parameter. For each of these positions, a different value of $G_{\mathrm{SET}}^{\mathrm{max}}$ or $G_{\mathrm{SET}}^{\min }$ is obtained by averaging the data over an extension of one quarter of a period (assuming sinusoidal oscillations, this would result in a visibility reduction smaller than $10 \%$ ). By separately extracting $G_{\mathrm{SET}}^{\mathrm{max} \text { min }}$ for the approximately ten periods, we calculate their mean values and estimate the corresponding standard errors. The error bars displayed Fig. $2 \mathrm{~b}$ are the standard error on the mean value of $\Delta Q$, obtained from the statistical uncertainty on $G_{\mathrm{SET}}^{\mathrm{max} \text { min }}$

Predictions in the quantum asymmetric regime. In the quantum asymmetric regime $\left(k_{\mathrm{B}} T \ll E_{\mathrm{C}}, \tau_{\mathrm{L}} \ll 1,1-\tau_{\mathrm{L}} \ll 1\right)$, the conductance reads (equation (34) in ref. 28 ):

$$
G_{\mathrm{SET}}^{\tau_{\mathrm{L}} \ll 1,1-\tau_{\mathrm{R}} \ll 1}=\tau_{\mathrm{L}} \frac{e^{2}}{h} \frac{2 \pi^{4}\left(k_{\mathrm{B}} T\right)^{2}}{3 \gamma^{2} E_{\mathrm{C}}^{2}} \times\left[1-2 \gamma \xi \sqrt{1-\tau_{\mathrm{R}}} \cos \left(2 \pi \delta V_{g} / \Delta\right)\right]
$$

with $\gamma \approx \exp (0.5772), \xi \approx 1.59, \Delta$ the gate-voltage period and $\delta V_{\mathrm{g}}$ the gatevoltage difference from charge degeneracy. In the ballistic limit $\left(1-\tau_{\mathrm{R}}=0\right)$, the conductance does not depend on gate voltage, but vanishes as $T^{2}$ following quantitatively, with the exact same pre-factor, the dynamical Coulomb blockade predictions ${ }^{2}$ for the same $E_{\mathrm{C}}$ and the corresponding series resistance $R=h / e^{2}$. Using equation (3), the visibility of the oscillations of conductance reads:

$$
\Delta Q\left(\tau_{\mathrm{L}} \ll 1,1-\tau_{\mathrm{R}} \ll 1\right)=2 \gamma \xi \sqrt{1-\tau_{\mathrm{R}}}
$$




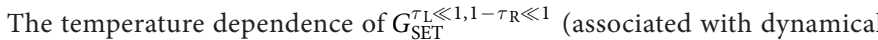
Coulomb blockade) cancels out in $\Delta Q$. Charge discreteness also affects the gate-voltage dependence of thermodynamic quantities, such as the average charge $(\langle Q\rangle)$ or the differential capacitance $\left(C_{\text {diff }} \equiv \partial\langle Q\rangle / \partial V_{\mathrm{g}}\right)$. The effect of Coulomb blockade on thermodynamic quantities was studied most comprehensively for tunnel junctions ${ }^{1,29}$ : at $T=0$ and $G \gg e^{2} / h$, the amplitude of average charge oscillations decays exponentially with $G h / e^{2}$; see, for example, refs $35-37$. The theoretical extension to multi-channel junctions of arbitrary transmission, beyond the tunnel limit, was performed in ref. 8 . In the presence of a single, nearly ballistic channel, the bosonization approach allows for an exact solution of the average charge in the metallic island in the low-energy 'quantum' regime $k_{\mathrm{B}} T \ll E_{\mathrm{C}}$ (equation (26) in ref. 7):

$$
\langle Q\rangle^{\tau_{\mathrm{L}} \ll 1,1-\tau_{\mathrm{R}} \ll 1}=\frac{e V_{\mathrm{g}}}{\Delta}-\frac{e \gamma}{\pi} \sqrt{1-\tau_{\mathrm{R}}} \sin \left(2 \pi V_{g} / \Delta\right)+Q_{0}
$$

with $Q_{0}$ a charge offset. In the ballistic limit $\left(1-\tau_{\mathrm{R}}=0\right)$, the charge increases linearly with gate voltage, corresponding to an absence of charge quantization. The degree of charge quantization can be characterized by the relative amplitude of the oscillations of charge or, equivalently, by the visibility of the differential capacitance $\left(C_{\text {diff }} \equiv \partial\langle Q\rangle / \partial V_{\mathrm{g}}\right)$ oscillations:

$$
\Delta C_{\mathrm{diff}}\left(\tau_{\mathrm{L}} \ll 1,1-\tau_{\mathrm{R}} \ll 1\right) \equiv \frac{C_{\mathrm{diff}}^{\max }-C_{\mathrm{diff}}^{\min }}{C_{\mathrm{diff}}^{\mathrm{max}}+C_{\mathrm{diff}}^{\min }}=2 \gamma \sqrt{1-\tau_{\mathrm{R}}}
$$

The degree of charge quantization vanishes as $\sqrt{1-\tau_{\mathrm{R}}}$ when approaching the ballistic limit, and does not depend on temperature in the quantum regime $\left(k_{\mathrm{B}} T \ll E_{\mathrm{C}}\right)$. Importantly, the visibility in the SET conductance oscillations is directly proportional to the visibility of the differential capacitance oscillations ${ }^{26}$, up to the fixed numerical factor $\xi \approx 1.59$ :

$$
\Delta Q\left(\tau_{\mathrm{L}} \ll 1,1-\tau_{\mathrm{R}} \ll 1\right)=\xi \Delta C_{\text {diff }}\left(\tau_{\mathrm{L}} \ll 1,1-\tau_{\mathrm{R}} \ll 1\right)
$$

Predictions in the quantum near-ballistic regime. In the quantum near-ballistic regime $\left(k_{\mathrm{B}} T \ll E_{\mathrm{C}}, 1-\tau_{\mathrm{L}, \mathrm{R}} \ll 1\right.$ ), the conductance $G_{\mathrm{SET}}$ reads (equations (38) and (26) in ref. 25):

$$
G_{\mathrm{SET}}=\frac{e^{2}}{2 h}\left[1-\int_{0}^{\infty} \frac{\Gamma_{-}^{2} / \cosh ^{2}(x)}{\left(x \pi^{2} k_{\mathrm{B}} T / \gamma E_{\mathrm{C}}\right)^{2}+\Gamma_{-}^{2}} \mathrm{~d} x\right]
$$

with $\gamma \approx \exp (0.5772)$ and

$$
\Gamma_{-}=\left(1-\tau_{\mathrm{L}}\right)+\left(1-\tau_{\mathrm{R}}\right)-2 \sqrt{\left(1-\tau_{\mathrm{L}}\right)\left(1-\tau_{\mathrm{R}}\right)} \cos \left(2 \pi \delta V_{\mathrm{g}} / \Delta\right)
$$

with $\Delta$ the gate-voltage period. The quantitative $\Delta Q$ predictions calculated with the maximum and minimum of $G_{\mathrm{SET}}$ inferred from equation (6) are displayed as coloured solid lines in Fig. 3. When approaching the ballistic critical point $\left(\sqrt{1-\tau_{\mathrm{L}, \mathrm{R}}} \ll k_{\mathrm{B}} T / E_{\mathrm{C}} \ll 1\right)$, the visibility $\Delta Q$ reduces to the simple asymptotic expression (equation (2) with $\gamma / \pi \approx 0.57$, reprinted here for convenience):

$$
\Delta Q\left(\sqrt{1-\tau_{\mathrm{L}, \mathrm{R}}} \ll \frac{k_{\mathrm{B}} T}{E_{\mathrm{C}}} \ll 1\right)=\frac{\gamma E_{\mathrm{C}}}{\pi k_{\mathrm{B}} T} \sqrt{\left(1-\tau_{\mathrm{L}}\right)\left(1-\tau_{\mathrm{R}}\right)}
$$

The differential capacitance $\left(C_{\text {diff }}\right)$ when one QPC approaches the ballistic critical point $\left(\tau_{\mathrm{R}} \rightarrow 1\right)$ reduces to the asymptotic expression (equation (41) in ref. 27):

$$
C_{\text {diff }}^{1-\tau_{\mathrm{R}} \ll 1-\tau_{\mathrm{L}} \ll 1}=-4 \gamma \frac{e}{\Delta} \ln \left(1-\tau_{\mathrm{L}}\right) \sqrt{\left(1-\tau_{\mathrm{L}}\right)\left(1-\tau_{\mathrm{R}}\right)} \cos \left(\frac{2 \pi \delta V_{\mathrm{g}}}{\Delta}\right)+\frac{e}{\Delta}
$$

and the visibility in the oscillations of the differential capacitance reads:

$$
\Delta C_{\text {diff }}\left(1-\tau_{\mathrm{R}} \ll 1-\tau_{\mathrm{L}} \ll 1\right)=-4 \gamma \ln \left(1-\tau_{\mathrm{L}}\right) \sqrt{\left(1-\tau_{\mathrm{L}}\right)\left(1-\tau_{\mathrm{R}}\right)}
$$

We recover the same $\sqrt{1-\tau_{\mathrm{R}}}$ scaling behaviour near the ballistic critical point $\left(\tau_{\mathrm{R}}=1\right)$ that was found in the asymmetric regime (equations (4) and (5)), and which is also found in the visibility of the conductance Coulomb oscillations (equation (7)). For two identical (for example, spin-degenerate) channels $\left(\tau \equiv \tau_{\mathrm{L}}=\tau_{\mathrm{R}}\right)$ near the ballistic critical point $(1-\tau \ll 1)$, the differential capacitance reads (equations (49) and (52) in ref. 7; a factor $e \Delta /\left(2 E_{\mathrm{C}}\right)$ was applied to match the definition $\left.C_{\text {diff }} \equiv \partial\langle Q\rangle / \partial V_{\mathrm{g}}\right)$ :

$$
\begin{aligned}
C_{\text {diff }}^{1-\tau \equiv 1-\tau_{\mathrm{L}}=1-\tau_{\mathrm{R}} \ll 1}= & \frac{4 \gamma e}{\pi \Delta} \ln \left[(1-\tau) \sin ^{2}\left(\frac{\pi \delta V_{\mathrm{g}}}{\Delta}\right)+\frac{k_{\mathrm{B}} T}{E_{\mathrm{C}}}\right] \\
& \times(1-\tau) \cos \left(\frac{2 \pi \delta V_{\mathrm{g}}}{\Delta}\right)+\frac{e}{\Delta}
\end{aligned}
$$

When approaching the ballistic critical point $(\tau \rightarrow 1)$, the visibility in the oscillations of the differential capacitance asymptotically vanishes as $1-\tau$, as in equation (7) with $\tau_{\mathrm{L}}=\tau_{\mathrm{R}}$.

Predictions in the presence of strong thermal fluctuations. In the presence of strong thermal fluctuations, $k_{\mathrm{B}} T \gg E_{\mathrm{C}} / \pi^{2}$, charge discreteness leads to periodic oscillations of the observables (for example, conductance and differential capacitance) while sweeping a capacitively coupled gate voltage. Quantum fluctuations decrease the oscillations, which are further attenuated by thermal fluctuations for increasing temperature, until the amplitude becomes exponentially small for $k_{\mathrm{B}} T \gg E_{\mathrm{C}} / \pi^{2}$. The exponential temperature dependence in $k_{\mathrm{B}} T / E_{\mathrm{C}}$ is quite robust, applying to thermodynamic ${ }^{1,8,29}$ and transport (Methods) properties. It can be demonstrated in the limits of both small and large transmission probabilities of the conduction channels comprising the junctions, and for various models of the metallic island. The presence of thermal fluctuations not only preserves the quantum $\sqrt{1-\tau}$ suppression of the oscillations, but it is expected from the results of ref. 8 that the square-root scaling of the differential capacitance extends with increasing temperature, up to the full range of $\tau_{\mathrm{L}, \mathrm{R}} \in[0,1]$. The relative oscillations in the differential capacitance and in the conductance characterize the degree of charge quantization equally well, both following the same $\exp \left(-\pi^{2} k_{\mathrm{B}} T / E_{\mathrm{C}}\right) \sqrt{\left(1-\tau_{\mathrm{L}}\right)\left(1-\tau_{\mathrm{R}}\right)}$ behaviour. Further information regarding the predictions and theoretical methods in the presence of strong thermal fluctuations are provided in the following four sections.

Differential capacitance in the tunnel limit with strong thermal fluctuations. This regime corresponds to $k_{\mathrm{B}} T \gg E_{\mathrm{C}} / \pi^{2}$ and $\tau_{\mathrm{L}, \mathrm{R}} \ll 1$. To start with, we evaluate the oscillatory part of the free energy of the island in the limit $\tau_{\mathrm{L}, \mathrm{R}} \ll 1$, where the suppression of charge quantization is entirely due to thermal fluctuations. Considering high temperatures, it is convenient to transform the partition function of the isolated island

$$
Z=\sum_{n=-\infty}^{\infty} \exp \left[-\frac{E_{n}(\mathcal{N})}{k_{\mathrm{B}} T}\right], \quad E_{n}(\mathcal{N})=E_{\mathrm{C}}(n-\mathcal{N})^{2}
$$

using the Poisson summation formula; the result is

$$
Z=\sqrt{\frac{\pi k_{\mathrm{B}} T}{E_{\mathrm{C}}}} \sum_{k=-\infty}^{\infty} \exp (-2 \pi i k \mathcal{N}) \exp \left(-\frac{\pi^{2} k_{\mathrm{B}} T}{E_{\mathrm{C}}} k^{2}\right)
$$

Here $\mathcal{N} \equiv V_{\mathrm{g}} / \Delta$ (with $\Delta$ the period in gate voltage $V_{\mathrm{g}}$ ) is the charge induced by the gate voltage in units of $e$, and the summations are performed over integers $n$ and $k$. The $k=0$ and $k= \pm 1$ terms in the sum in equation (8) yield, respectively, the leading $\mathcal{N}$-independent and $\mathcal{N}$-dependent contributions $F_{0}$ and $\delta F(\mathcal{N})$ to the free energy $F=-k_{\mathrm{B}} T \ln (Z)$ at $k_{\mathrm{B}} T \gg E_{\mathrm{C}} / \pi^{2}$. The resulting oscillatory part of the differential capacitance,

$C_{\mathrm{diff}}^{\tau_{\mathrm{L}, \mathrm{R}} \ll 1} \equiv \frac{e}{\Delta}\left(1-\frac{1}{2 E_{\mathrm{C}}} \partial_{\mathcal{N}}^{2} F\right)=\frac{e}{\Delta}-4 \frac{e}{\Delta} \frac{\pi^{2} k_{\mathrm{B}} T}{E_{\mathrm{C}}} \exp \left(-\frac{\pi^{2} k_{\mathrm{B}} T}{E_{\mathrm{C}}}\right) \cos (2 \pi \mathcal{N})$

is exponentially suppressed at high temperatures.

Differential capacitance in the near-ballistic regime with strong thermal fluctuations. This regime corresponds to $k_{\mathrm{B}} T \gg E_{\mathrm{C}} / \pi^{2}$ and $1-\tau_{\mathrm{R}} \ll 1$. A similar suppression of oscillations of the thermodynamic characteristics can also be demonstrated in the case of high-transmission junctions, where both thermal and quantum fluctuations contribute to the reduction of charge quantization. For definiteness, we consider here a single-junction case $\left(\tau_{\mathrm{L}}=0\right)$ with $1-\tau_{\mathrm{R}} \ll 1$. Evaluation of $C_{\text {diff }}^{\tau_{\mathrm{L}}=0,1-\tau_{\mathrm{R}} \ll 1}$ can be performed using the bosonization scheme developed in ref. 7. In that formalism, the $\mathcal{N}$-dependent part of the differential capacitance reads

$$
\delta C_{\text {diff }}^{\tau_{\mathrm{L}}=0,1-\tau_{\mathrm{R}} \ll 1}=-\frac{e}{\Delta} \frac{2 \pi^{2}}{E_{\mathrm{C}}} D \sqrt{1-\tau_{\mathrm{R}}}\langle\cos [2 \pi \mathcal{N}-\varphi(0)]\rangle
$$

where the bosonic quantum field $\varphi(0)=2 \pi \hat{Q} / e$ corresponds to the charge $\hat{Q}$ passed through the junction $(x=0)$, and $D$ is the energy bandwidth appearing in the definition of boson variables. Averaging $\langle\ldots\rangle$ is performed over the fluctuations of the field $\varphi(x)$. The Hamiltonian describing these fluctuations consists of two parts ${ }^{7}$, representing the energy of particle-hole excitations and the charging energy, respectively. The former part depends on $(\nabla \varphi)^{2}$ and the latter part has the form $E_{C}[\varphi(0) /(2 \pi)]^{2}$. Replacement of the ground-state averaging ${ }^{7}$ with an average over the Gibbs distribution of fluctuations, which is proportional to $\exp \left\{-\left[E_{\mathrm{C}} /\left(k_{\mathrm{B}} T\right)\right]\right.$ $\left.[\varphi(0) /(2 \pi)]^{2}\right\}$, results in the renormalization of the bandwidth $D$ to a physically meaningful value of approximately $k_{\mathrm{B}} T$ and in exponential suppression of the oscillations at $k_{\mathrm{B}} T \gg E_{\mathrm{C}} / \pi^{2}$. 


$$
\delta C_{\text {diff }}^{\tau_{\mathrm{L}}=0,1-\tau_{\mathrm{R}} \ll 1} \approx-4 \frac{e}{\Delta} \frac{\pi^{2} k_{\mathrm{B}} T}{E_{\mathrm{C}}} \exp \left(-\frac{\pi^{2} k_{\mathrm{B}} T}{E_{\mathrm{C}}}\right) \sqrt{1-\tau_{\mathrm{R}}} \cos (2 \pi \mathcal{N})
$$

Because it follows from ref. 8, equation (10) is applicable in the full range of $\tau_{\mathrm{R}}$ for $k_{\mathrm{B}} T \gg E_{\mathrm{C}} / \pi^{2}$ (the numerical coefficient in equation (10) was established with the help of ref. 8). The identical exponential suppression for an almost-isolated island (equation (9)) is therefore simply equation (10) in the limit $\tau_{\mathrm{R}} \ll 1$. In addition, quantum fluctuations contribute to the same suppression factor $\sqrt{1-\tau_{\mathrm{R}}}$ derived at $1-\tau_{\mathrm{R}} \ll 1$ in the quantum regime $k_{\mathrm{B}} T \ll E_{\mathrm{C}}$ (equation (5)). Furthermore, equation (10) derived for $k_{\mathrm{B}} T \gg E_{\mathrm{C}}$ matches the $T=0$ result of ref. 7 at $k_{\mathrm{B}} T \approx E_{\mathrm{C}}$; however, given the large numerical factor $\pi^{2}$ in the exponent of equation (10), there may be a broad crossover temperature region between the two limits.

Conductance in the tunnel limit with strong thermal fluctuations. This regime corresponds to $k_{\mathrm{B}} T \gg E_{\mathrm{C}} / \pi^{2}$ and $\tau_{\mathrm{L}, \mathrm{R}} \ll 1$. Turning now to conductance oscillations, we again start from the simpler case of low-transmission barriers $\left(\tau_{\mathrm{L}, \mathrm{R}} \ll 1\right)$. In that limit, the rate equation for current carried by spin-polarized electrons yields ${ }^{38}$.

$$
\begin{aligned}
& G_{\mathrm{SET} T}^{\tau_{\mathrm{L}, \mathrm{R}} \ll 1}(\mathcal{N}, T) \\
& \quad=\frac{e^{2}}{h} \frac{\tau_{\mathrm{L}} \tau_{\mathrm{R}}}{\tau_{\mathrm{L}}+\tau_{\mathrm{R}}} \sum_{n=-\infty}^{\infty}\left\{\frac{\exp \left[-E_{n}(\mathcal{N}) /\left(k_{\mathrm{B}} T\right)\right]}{Z(\mathcal{N}, T)} \times f\left(\frac{E_{n}(\mathcal{N})-E_{n-1}(\mathcal{N})}{k_{\mathrm{B}} T}\right)\right\}
\end{aligned}
$$

where $f(x)=x /\left(1-\mathrm{e}^{-x}\right)$. Application of the Poisson summation formula to the above equation is tedious, but straightforward. The result is an expression for $G_{\mathrm{SET}}^{\tau \mathrm{L}, \mathrm{R}} \ll 1$ that involves a sum of harmonics proportional to $\cos (2 \pi k \mathcal{N})$, similar to equation (8). The largest term,

$$
G_{\infty}=\frac{e^{2}}{h}\left(\frac{1}{\tau_{\mathrm{L}}}+\frac{1}{\tau_{\mathrm{R}}}\right)^{-1}
$$

does not oscillate and is simply the conductance of two resistors connected in series. The leading oscillatory term,

$$
\delta G_{\mathrm{SET}}^{\tau \mathrm{L}, \mathrm{R}} \ll 1(\mathcal{N}, T)=-G_{\infty} \frac{\pi^{2} k_{\mathrm{B}} T}{E_{\mathrm{C}}} \exp \left(-\frac{\pi^{2} k_{\mathrm{B}} T}{E_{\mathrm{C}}}\right) \cos (2 \pi \mathcal{N})
$$

exhibits the same exponential suppression as the differential capacitance (equation (9)).

Conductance in the near-ballistic regime with strong thermal fluctuations. This regime corresponds to $k_{\mathrm{B}} T \gg E_{\mathrm{C}} / \pi^{2}$ and $1-\tau \ll 1$. Regarding the conductance across a metallic island with high-transmission contacts, we (A) present a formalism that is somewhat different from ref. 25 , details of which will be published separately (E.I., I.P.L. and E.V.S., manuscript in preparation) and (B) further establish the predictions by extending the formalism of ref. 25 to high temperatures.

(A) In the first approach, we start from the chiral edge excitations of the integer quantum Hall regime, in close correspondence with the experimental configuration. Although we are interested in the high-temperature limit, all the energy scales in the experiment remain much smaller than the quantum Hall energy gap. At such low energies, the quantum Hall edge states may be described by the effective theor ${ }^{39-41}$. According to this theory, edge excitations can be viewed as bosonic edge magneto-plasmons. The corresponding one-dimensional charge density waves $\rho_{s \alpha}(x)(s \in\{\mathrm{L}, \mathrm{R}\}, \alpha \in\{1,2\}$; see Extended Data Fig. 4 for notations) verify the canonical commutation relations $\left[\rho_{s \alpha}(x), \rho_{s \prime \beta}(y)\right]=(-1)^{\alpha} 2 \pi i e^{2} \delta_{s s} \delta_{\alpha \beta} \delta^{\prime}(x-y)$, where the sign accounts for the propagation direction of the chiral edge states, $\delta_{s s^{\prime}}$ and $\delta_{\alpha \beta}$ are Kronecker delta functions, and $\delta^{\prime}(x-y)$ is the derivative of the Dirac delta function.

The Hamiltonian of the experimental set-up contains three terms: $\mathcal{H}=\mathcal{H}_{0}+\mathcal{H}_{\text {int }}+\mathcal{H}_{\mathrm{T}}$. The first term describes the dynamics of the bare edge states:

$$
\mathcal{H}_{0}=\frac{h v_{\mathrm{F}}}{2 e^{2}} \sum_{s \alpha} \int \mathrm{d} x \rho_{s \alpha}^{2}(x)
$$

where $v_{\mathrm{F}}$ is the Fermi velocity of the quantum Hall edge states. The second term describes Coulomb interactions at the metallic island:

$$
\begin{aligned}
\mathcal{H}_{\text {int }} & =E_{\mathrm{C}}(\hat{Q} / e-\mathcal{N})^{2} \\
\hat{Q}=\frac{e}{2 \pi}\left[\varphi_{\mathrm{L}}(0)-\varphi_{\mathrm{R}}(0)\right] & =\sum_{\alpha}\left[\int_{0}^{\infty} \mathrm{d} x \rho_{\mathrm{L} \alpha}(x)+\int_{-\infty}^{0} \mathrm{~d} x \rho_{\mathrm{R} \alpha}(x)\right]
\end{aligned}
$$

The first equality in equation (13) defines the Bose field operators that are also used in the derivation of equation (10), but here for the case of two contacts. The last term in the Hamiltonian describes the backscattering of electrons at the two QPCs:

$$
\begin{gathered}
\mathcal{H}_{\mathrm{T}}=A_{\mathrm{L}}+A_{\mathrm{R}}+\text { h.c. } \\
A_{s}=\gamma_{s} \psi_{s 1}^{\dagger}(0) \psi_{s 2}(0) \\
\psi_{s \alpha}(0)=\sqrt{\frac{D}{h v_{\mathrm{F}}}} \exp \left[\frac{2 \pi i}{e} \int_{-\infty}^{0} \mathrm{~d} x \rho_{s \alpha}(x)\right]
\end{gathered}
$$

where the backscattering amplitudes $\gamma_{\mathrm{L}, \mathrm{R}}$ depend on the 'intrinsic' transmission probabilities $\tau_{\mathrm{L}, \mathrm{R}}$ (in the near ballistic regime, $1-\tau_{\mathrm{L}(\mathrm{R})} \approx\left|\gamma_{\mathrm{L}(\mathrm{R})}\right|^{2} /\left(\hbar v_{\mathrm{F}}\right)^{2}$.

We set the distance between the metallic island and the QPCs, which is much shorter than the wavelength of excitations in the experiment, to zero. We stress that exactly the same Hamiltonian arises in the absence of the quantum Hall effect, when applying the bosonization procedure to a metallic island connected to reservoirs through spin-polarized electron channels (as in refs 7 and 25). Consequently, the predictions below apply beyond the quantum Hall configuration used here as a starting point.

Focusing on the near ballistic regime $1-\tau_{\mathrm{L}, \mathrm{R}} \ll 1$, we apply the scattering theory approach developed in refs 42 and 43 . The average $\langle I\rangle \equiv \operatorname{tr}(\rho I)$ of the current operator $I=v_{F}\left[\rho_{\mathrm{R} 1}(0)-\rho_{\mathrm{R} 2}(0)\right]$ is evaluated perturbatively in backscattering amplitudes (equation (14)). With this aim, we express the density matrix $\rho=U \rho_{0} U^{\dagger}$ in terms of its equilibrium value $\rho_{0} \propto \exp \left[-\left(\mathcal{H}_{0}+\mathcal{H}_{\text {int }}\right) /\left(k_{\mathrm{B}} T\right)\right]$, and expand the evolution operator $U=\hat{T} \exp \left[-2 \pi i / h \int \mathrm{d} t H_{\mathrm{T}}(t)\right]$ in powers of $\gamma_{s}$, where $\hat{T} \exp$ indicates the time-ordered exponential. This results in the two leading terms:

$$
\langle I\rangle=\langle I\rangle_{0}+\frac{1}{\hbar^{2}}\left\langle\iint \mathrm{d} t^{\prime} \mathrm{d} t^{\prime \prime}\left\{\mathcal{H}_{\mathrm{T}}\left(t^{\prime}\right),\left[\mathcal{H}_{\mathrm{T}}\left(t^{\prime \prime}\right), I\right]\right\}\right\rangle_{0}
$$

where the average is taken with respect to the equilibrium density matrix $\rho_{0}$.

The Hamiltonian $\mathcal{H}_{0}+\mathcal{H}_{\text {int }}$ is quadratic in plasmon operators. Consequently, the corresponding dynamics can be accounted for exactly within the scattering theory approach for bosons $\mathrm{s}^{42,43}$. For instance, the scattering matrix for the interaction Hamiltonian $\mathcal{H}_{\text {int }}$ (ignoring the backscattering Hamiltonian $\mathcal{H}_{\mathrm{T}}$ ), which relates the currents in the incoming (L1, R2, L2, R1) and outgoing (L2, R1, L1, R2) channels at the frequency $\omega /(2 \pi)$, reads:

$$
S(\omega)=\frac{1}{2}\left(\begin{array}{cccc}
z & z & 2-z & -z \\
z & z & -z & 2-z \\
2-z & -z & z & z \\
-z & 2-z & z & z
\end{array}\right)
$$

where $z=1 /\left[i h \omega /\left(4 E_{C}\right)+1\right]$. Taking the limit $\omega \rightarrow 0$ (ref. 43), we determine the first term in equation (16): $\langle I\rangle_{0}=e^{2} V_{\mathrm{dc}} /(2 h)$. The bare conductance is thus half the conductance quantum. In the limit of small d.c. bias $V_{\mathrm{dc}}$, the second term can be rewritten as

$$
\delta\langle I\rangle=\frac{e}{\hbar^{2}} \int \mathrm{d} t\left\langle\left[A_{\mathrm{L}}^{\dagger}(t)+A_{\mathrm{R}}^{\dagger}(t), A_{\mathrm{L}}(0)+A_{\mathrm{R}}(0)\right]\right\rangle_{0}
$$

This term contains the coherent contribution

$$
\delta\langle I\rangle_{\mathrm{osc}}=\frac{e}{\hbar^{2}} \operatorname{Re} \gamma_{\mathrm{L}}^{*} \gamma_{\mathrm{R}} \int \mathrm{d} t\left\langle\psi_{\mathrm{L} 2}^{\dagger}(0, t) \psi_{\mathrm{L} 1}(0, t) \psi_{\mathrm{R} 1}^{\dagger}(0,0) \psi_{\mathrm{R} 2}(0,0)\right\rangle_{0}
$$

which oscillates as a function of the induced charge $e \mathcal{N}$.

In general, one can use the scattering matrix equation (17) to evaluate the average in equation (18), which leads to a complex expression (E.I., I.P.L. and E.V.S., manuscript in preparation). However, the leading high-temperature asymptotics can be found using exactly the same argument as for the case of the differential capacitance considered above. Specifically, according to equation (15), the particular value of the charge $Q$ in the island leads to the phase shift of $\exp [2 \pi i(Q / e-\mathcal{N})]$ in the correlation function in equation (18). Therefore, by averaging the correlation function over instant fluctuations of this charge, which are distributed with the equilibrium Gibbs weights (proportional to $\exp \left[-(Q / e)^{2} E_{\mathrm{C}} /\left(k_{\mathrm{B}} T\right)\right]$ ), we determine the high-temperature behaviour of the oscillating part of the current:

$$
\begin{aligned}
\delta\langle I\rangle_{\mathrm{osc}} & \propto \frac{e^{2} V_{\mathrm{dc}}}{h} \sqrt{\frac{E_{\mathrm{C}}}{k_{\mathrm{B}} T}} \sqrt{\left(1-\tau_{\mathrm{L}}\right)\left(1-\tau_{\mathrm{R}}\right)} \\
& \times \int \mathrm{d} Q \exp \left(-\frac{Q^{2} E_{\mathrm{C}}}{e^{2} k_{\mathrm{B}} T}\right) \cos [2 \pi(\mathcal{N}-Q / e)] \\
& \propto \frac{e^{2} V_{\mathrm{dc}}}{h} \exp \left(-\frac{\pi^{2} k_{\mathrm{B}} T}{E_{\mathrm{C}}}\right) \sqrt{\left(1-\tau_{\mathrm{L}}\right)\left(1-\tau_{\mathrm{R}}\right)} \cos (2 \pi \mathcal{N})
\end{aligned}
$$

The validity of this simplified approach is confirmed by calculations (E.I., I.P.L. and E.V.S., manuscript in preparation). 
(B) An alternative route of calculation amounts to re-working equation (A5) of ref. 25 for the case $1-\tau_{\mathrm{L}, \mathrm{R}} \ll 1$ or equation (A27) for the asymmetric case $\tau_{\mathrm{L}} \ll 1$, $1-\tau_{\mathrm{R}} \ll 1$. In either case, the largest term in the limit $k_{\mathrm{B}} T \gg E_{\mathrm{C}} / \pi^{2}$ is, unsurprisingly, $\mathcal{N}$-independent. Like equation (11), it represents the conductance of two junctions connected in series: $G_{\infty} \approx e^{2} /(2 h)$ in the case of $1-\tau_{\mathrm{L}, \mathrm{R}} \ll 1$ and $G_{\infty} \approx\left(e^{2} / h\right) \tau_{\mathrm{L}}$ in the case $\tau_{\mathrm{L}} \ll 1,1-\tau_{\mathrm{R}} \ll 1$. The leading oscillatory term in the former case is

$$
\delta G_{\mathrm{SET}}^{1-\tau_{\mathrm{L}, \mathrm{R}} \ll 1}(\mathcal{N}, T) \approx \frac{e^{2}}{h} \exp \left(-\frac{\pi^{2} k_{\mathrm{B}} T}{E_{\mathrm{C}}}\right) \sqrt{\left(1-\tau_{\mathrm{L}}\right)\left(1-\tau_{\mathrm{R}}\right)} \cos (2 \pi \mathcal{N})
$$

In the asymmetric case, the factor $\sqrt{\left(1-\tau_{\mathrm{L}}\right)\left(1-\tau_{\mathrm{R}}\right)}$ in the above expression is replaced by $\tau_{\mathrm{L}} \sqrt{1-\tau_{\mathrm{R}}}$. The visibility of conductance oscillations now reads:

$$
\Delta Q \approx \exp \left(-\frac{\pi^{2} k_{\mathrm{B}} T}{E_{\mathrm{C}}}\right) \sqrt{\left(1-\tau_{\mathrm{L}}\right)\left(1-\tau_{\mathrm{R}}\right)}
$$

This form correctly extrapolates between the symmetric and asymmetric cases. Conductance at $T \approx 17 \mathrm{mK}$ versus quantum regime predictions. Although the visibility $\Delta Q$ of the oscillations in the SET conductance best reflects the degree of charge quantization, we can also confront experiment and theory directly at the underlying conductance-sweeps level. In Extended Data Fig. 3, we compare $G_{\text {SET }}\left(\delta V_{\mathrm{g}}\right)$ measurements (symbols) and predictions near the ballistic critical point $\left(1-\tau_{\mathrm{R}} \approx 0.02\right.$ and 0.004$)$ with $\mathrm{QPC}_{\mathrm{L}}$ in both the tunnel $\left(\tau_{\mathrm{L}}=0.075\right)$ and almost perfectly transmitted $\left(1-\tau_{\mathrm{L}} \approx 0.02\right)$ regimes. Solid lines are calculated with the electronic temperature $T=17 \mathrm{mK}$, using equation (3) for the top two panels (asymmetric regime, $\tau_{\mathrm{L}}=0.075$ ) and equation (6) for the bottom two panels (near ballistic regime, $\tau_{\mathrm{L}}=0.983$ ). The grey areas correspond to the experimental uncertainty of $\pm 4 \mathrm{mK}$. The demonstrated agreement validates the full prediction for the renormalized SET conductance.

Charge quantization based on conductance or transmission probability values. Theory predicts that as soon as one conduction channel connected to the metallic island is ballistic, the charge in the island is completely unquantized. Here we show that charge quantization collapses systematically at the ballistic critical point $\tau_{\mathrm{R}}=1$, independent of the setting of the second channel $\left(\tau_{\mathrm{L}}<1\right)$. We further demonstrate that the crucial ingredient is not the overall conductance, but the presence of a perfectly transmitted channel. For this purpose, we compare the two configurations displayed in Extended Data Fig. 5a, b. In both configurations, $\mathrm{QPC}_{\mathrm{L}}$ is tuned to the same standard setting corresponding to a single conduction channel of 'intrinsic' transmission probability $\tau_{\mathrm{L}}=0.24$. In both configurations, $\mathrm{QPC}_{\mathrm{R}}$ is set to the same overall intrinsic conductance $G_{\mathrm{R}}^{\mathrm{qpc}} \equiv \tau_{\mathrm{R}} e^{2} / h=1.5 e^{2} / h$. However, in the first configuration (Extended Data Fig. 5a) $\mathrm{QPC}_{\mathrm{R}}$ decomposes into one ballistic channel and one channel of intrinsic transmission probability 0.5 , whereas in the second configuration (Extended Data Fig. 5b) it decomposes into two non-ballistic channels of intrinsic transmission probabilities 0.7 and 0.8. (In practice, the $Q P C_{R}$ of the second configuration is realized using two different physical QPCs biased at the same voltage.) As shown Extended Data Fig. 5c, the SET conductance displays strong oscillations in the second configuration, signalling charge quantization in the absence of a ballistic channel. By contrast, the SET conductance in the first configuration does not depend on gate voltage, signalling a completely unquantized island charge in the presence of one ballistic channel.

31. Göktas, O., Weber, J., Weis, J. \& von Klitzing, K. Alloyed ohmic contacts to two-dimensional electron system in $\mathrm{AIGaAs} / \mathrm{GaAs}$ heterostructures down to submicron length scale. Physica E 40, 1579-1581 (2008).

32. Iftikhar, Z. et al. Two-channel Kondo effect and renormalization flow with macroscopic quantum charge states. Nature 526, 233-236 (2015).

33. Jezouin, S. et al. Quantum limit of heat flow across a single electronic channel. Science 342, 601-604 (2013).

34. Spietz, L., Lehnert, K. W., Siddiqi, I. \& Schoelkopf, R. J. Primary electronic thermometry using the shot noise of a tunnel junction. Science $\mathbf{3 0 0}$, 1929-1932 (2003).

35. Panyukov, S. V. \& Zaikin, A. D. Coulomb blockade and nonperturbative ground-state properties of ultrasmall tunnel junctions. Phys. Rev. Lett. 67, 3168-3171 (1991).

36. Wang, X. \& Grabert, H. Coulomb charging at large conduction. Phys. Rev. B 53, 12621-12624 (1996).

37. Lukyanov, S. L. Notes on parafermionic QFTs with boundary interaction. Nucl. Phys. B 784, 151-201 (2007).

38. Glazman, L. I. \& Shekhter, R. I. Coulomb oscillations of the conductance in a laterally confined heterostructure. J. Phys. Condens. Matter 1, 5811-5815 (1989).

39. Halperin, B. I. Quantized Hall conductance, current-carrying edge states, and the existence of extended states in a two-dimensional disordered potential. Phys. Rev. B 25, 2185-2190 (1982).

40. Wen, X. G. Chiral Luttinger liquid and the edge excitations in the fractional quantum Hall states. Phys. Rev. B 41, 12838-12844 (1990).

41. Fröhlich, J. \& Zee, A. Large scale physics of the quantum Hall fluid. Nucl. Phys. B 364, 517-540 (1991).

42. Slobodeniuk, A., Levkivskyi, I. \& Sukhorukov, E. Equilibration of quantum Hall edge states by an Ohmic contact. Phys. Rev. B 88, 165307 (2013).

43. Sukhorukov, E. Scattering theory approach to bosonization of non-equilibrium mesoscopic systems. Physica E 77, 191-198 (2016). 


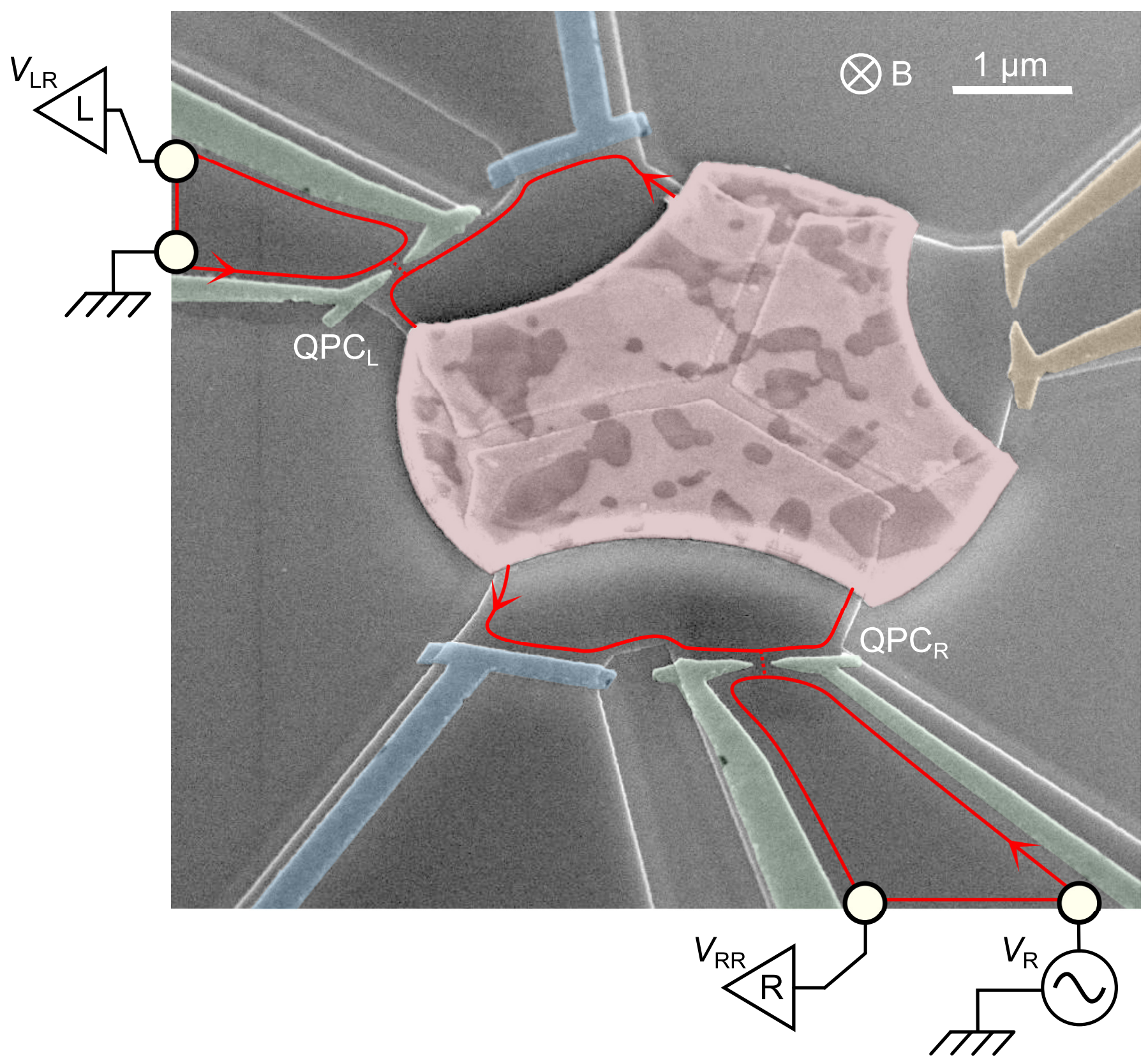

Extended Data Figure $1 \mid$ Measurement schematic. The signal $V_{\mathrm{LR}}\left(V_{\mathrm{RR}}\right)$ is the voltage measured with amplification chain $\mathrm{L}(\mathrm{R})$ in response to the injected voltage $V_{\mathrm{R}}$. The trenches etched in the 2DEG, which can be seen in the form of a ' $Y$ ' through the metallic island, ensure that the only way from one QPC to the other is across the metallic island. The experiment is performed in the quantum Hall regime at filling factor $\nu=2$, where the current propagates along the edges in the direction indicated by arrows. 

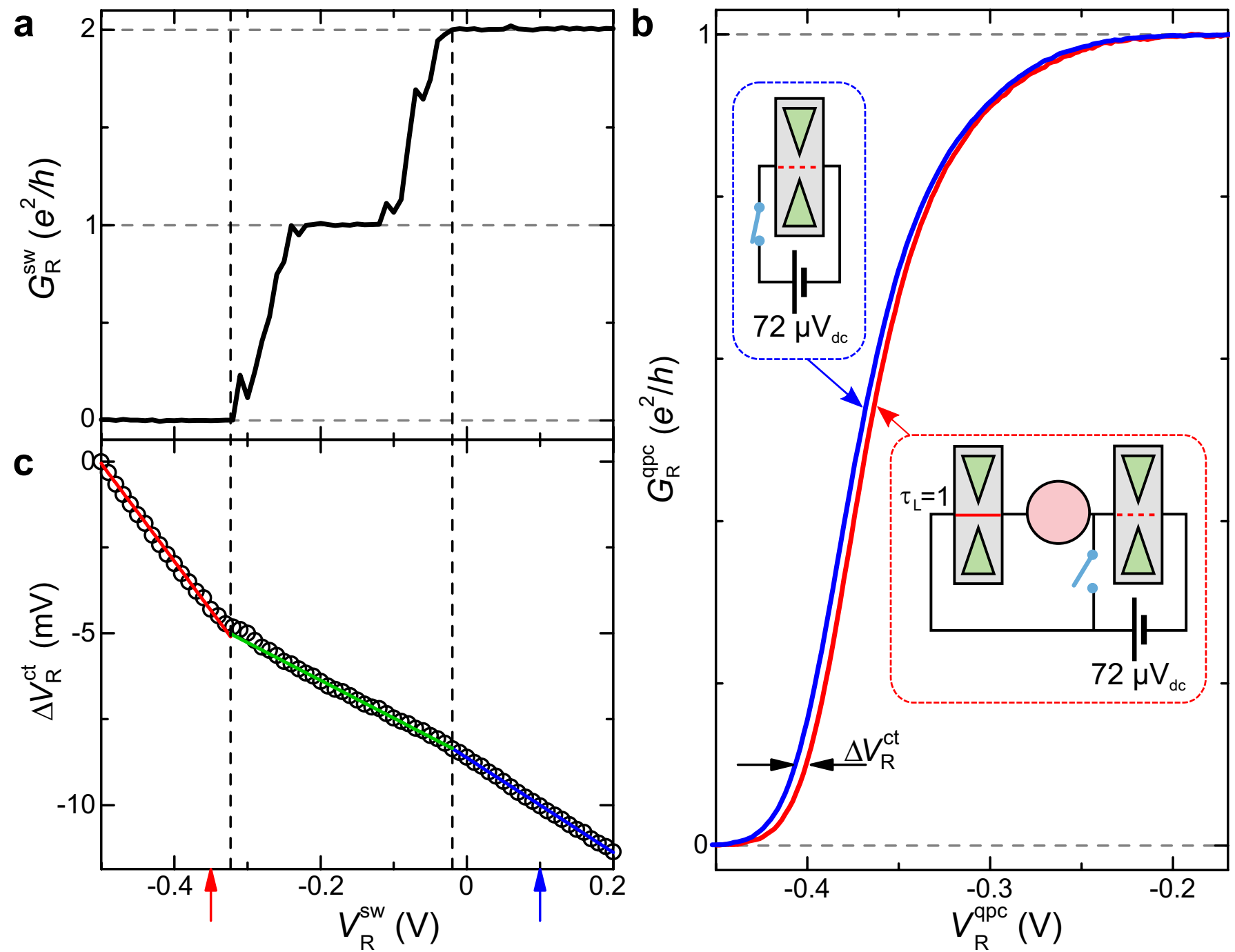

Extended Data Figure 2 $\mid$ Crosstalk compensation. a, (Intrinsic) conductance $G_{\mathrm{R}}^{\mathrm{sw}}$ across the characterization gate adjacent to $\mathrm{QPC}_{\mathrm{R}}$ versus gate voltage $V_{\mathrm{R}}^{\mathrm{sW}}$. In the experiment, the left and right switches are independently set to the open and closed positions with $V_{\mathrm{R}, \mathrm{L}}^{\mathrm{sw}}=-0.35 \mathrm{~V}$ and $V_{\mathrm{R}, \mathrm{L}}^{\mathrm{sW}}=0.1 \mathrm{~V}$, respectively (vertical arrows in $\mathbf{c}$ ). $\mathbf{b}, \mathrm{QPC}_{\mathrm{R}}$ differential conductance in the presence of a d.c. bias of $72 \mu \mathrm{V}\left({ }^{\prime} 72 \mu \mathrm{V}_{\mathrm{dc}}{ }^{\prime}\right)$ versus $\mathrm{QPC}$ gate voltage $V_{\mathrm{R}}^{\mathrm{qpc}}$. The red and blue lines are measured with the adjacent switch in the open and closed positions, respectively (see inset schematics). The voltage drop across $\mathrm{QPC}_{\mathrm{R}}$ is smaller with the switch

open, owing to the added series resistance. Although this does not result in a large error, because $G_{\mathrm{R}}^{\mathrm{qpc}}$ depends weakly on voltage bias, this effect is minimized by extracting the crosstalk compensation $\Delta V_{\mathrm{R}}^{\mathrm{ct}}$ at low $G_{\mathrm{R}}^{\mathrm{qpc}} \lesssim 0.1 e^{2} / h$. c, Symbols represent the crosstalk compensation $\Delta V_{\mathrm{R}}^{\mathrm{ct}}$, with respect to the gate voltage $V_{\mathrm{R}}^{\mathrm{sW}}=-0.5 \mathrm{~V}$, versus $V_{\mathrm{R}}^{\mathrm{sw}}$. Lines are linear fits of the crosstalk compensation at $G_{\mathrm{R}}^{\mathrm{sw}}=0$ (red, $-2.8 \%$ relative compensation), $0<G_{\mathrm{R}}^{\mathrm{sw}}<2 e^{2} / h$ (green, $-1.1 \%$ relative compensation) and $G_{\mathrm{R}}^{\mathrm{sw}}=2 e^{2} / h$ (blue, $-1.4 \%$ relative compensation). 


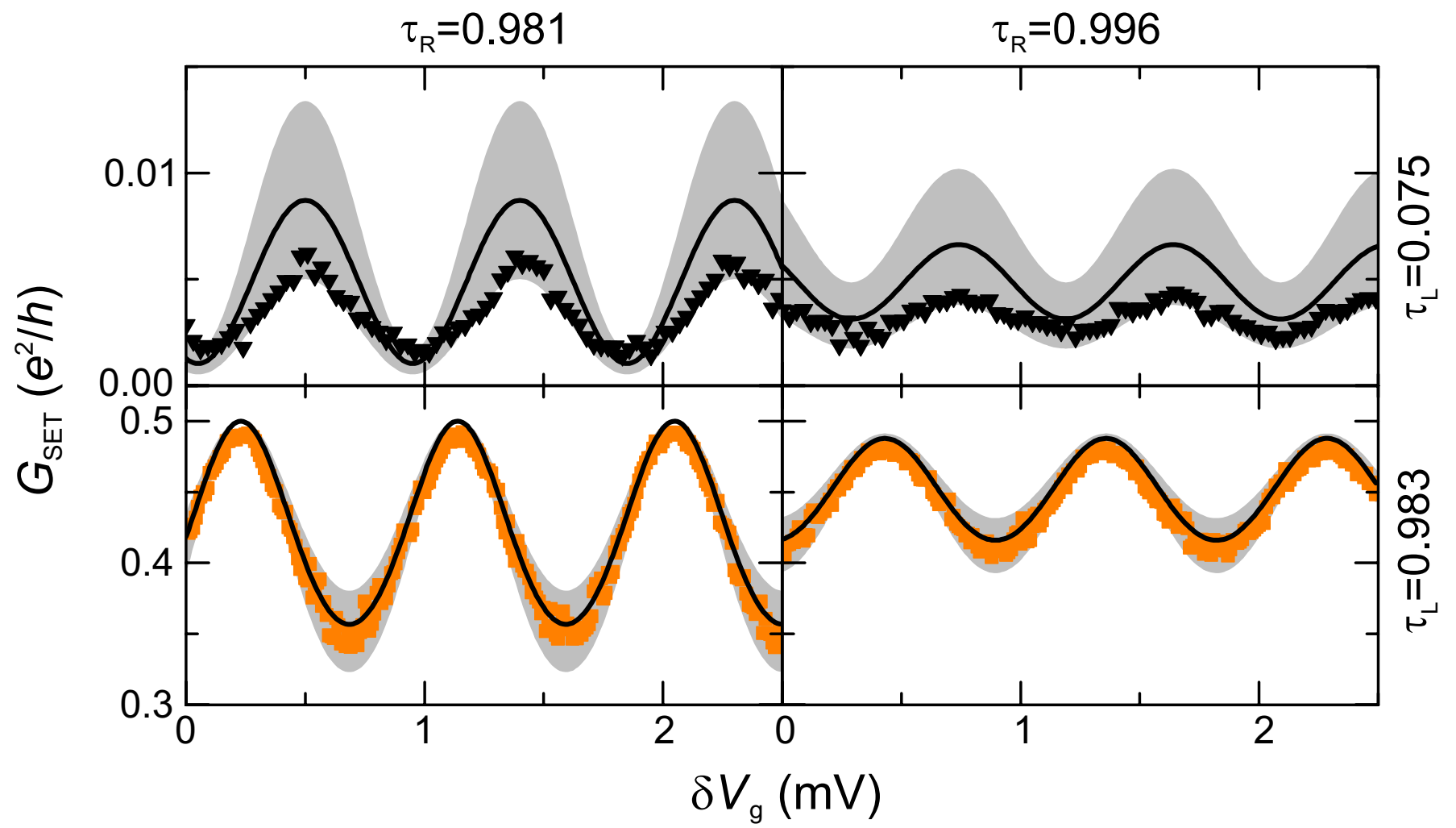

Extended Data Figure 3 | Conductance measurements versus quantitative predictions. Direct $G_{\mathrm{SET}}\left(\delta V_{\mathrm{g}}\right)$ comparison at $T \approx 17 \mathrm{mK}$ between data (symbols) and predictions (solid lines, grey areas correspond to the temperature uncertainty of $\pm 4 \mathrm{mK}$ ) in the two limits addressed by theory (equation (3) for $\tau_{\mathrm{L}} \approx 0$ (top panels), equation (6) for $\tau_{\mathrm{L}} \approx 1$ (bottom panels)). 


\section{RESEARCH LETTER}

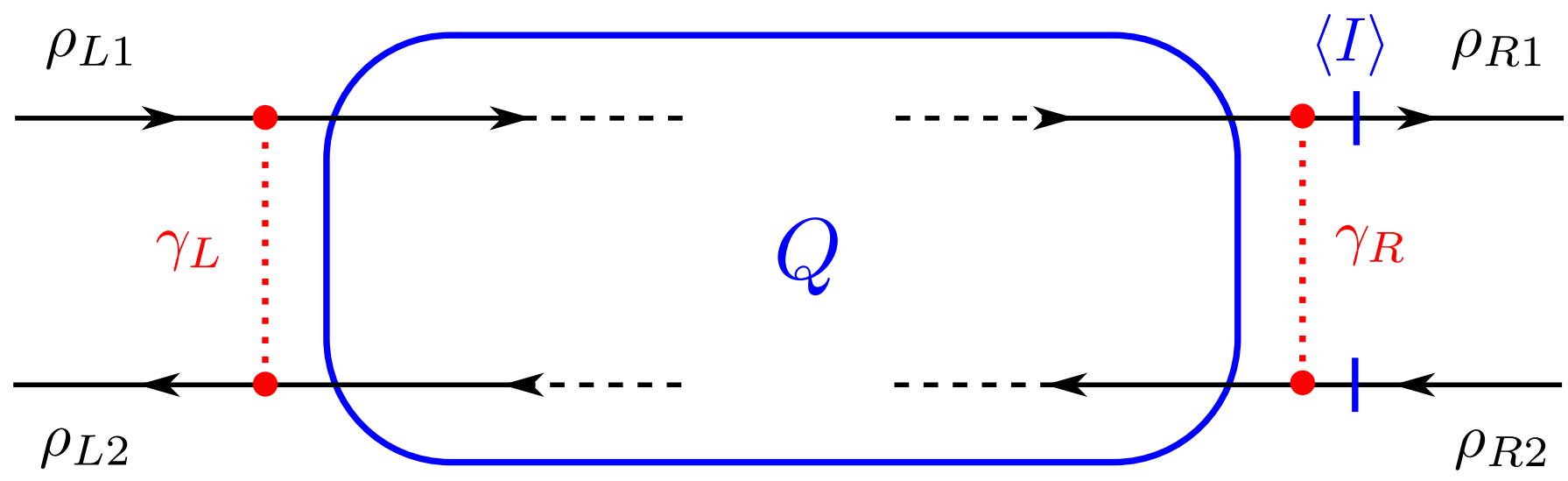

Extended Data Figure 4 | Theoretical description of the experimental set-up in formalism (A) for strong thermal fluctuations. We consider the regime of the quantum Hall effect, where only one spinless edge mode contributes to the transport. The corresponding edge states are described by four charge density operators, labelled by $s \in\{L, R\}$ and $\alpha \in\{1,2\}$.

These states are mixed (backscattered) at the two QPCs (red dashed lines)

with amplitudes $\gamma_{\mathrm{L}}$ and $\gamma_{\mathrm{R}}$ (equations (14) and (15)). The edge densities enter into the interaction Hamiltonian (equation (12) through the total charge $\hat{Q}$ of the metallic island (equation (13)). The average current $\langle I\rangle$ is calculated through a cross-section immediately to the right of $\mathrm{QPC}_{R}$ (vertical blue lines). 
a

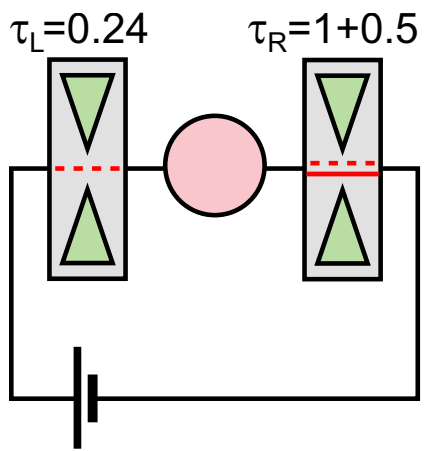

b

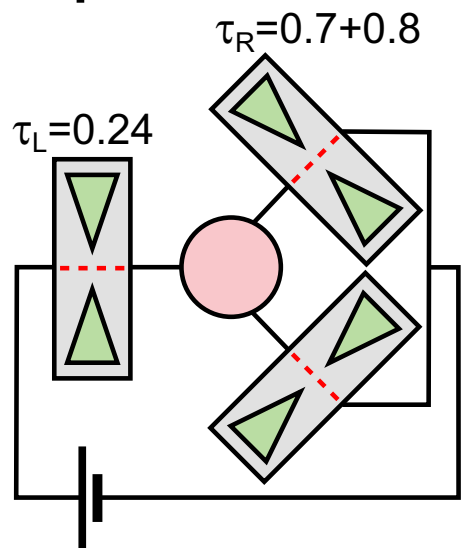

Extended Data Figure 5 | Charge quantization based on conductance versus transmission probability values. $\mathbf{a}, \mathbf{b}$, Schematics of the configurations, both with the same $\mathrm{QPC}_{\mathrm{L}}$ setting $\tau_{\mathrm{L}}=0.24$. In the configuration shown in a, $\mathrm{QPC}_{\mathrm{R}}$ is set to an 'intrinsic' conductance $G_{\mathrm{R}}^{\mathrm{qpc}} \equiv \tau_{\mathrm{R}} e^{2} / h=1.5 e^{2} / h$, which decomposes into one ballistic channel and one channel of intrinsic transmission probability 0.5 . In the configuration shown in $\mathbf{b}, \mathrm{QPC}_{\mathrm{R}}$ is set

C

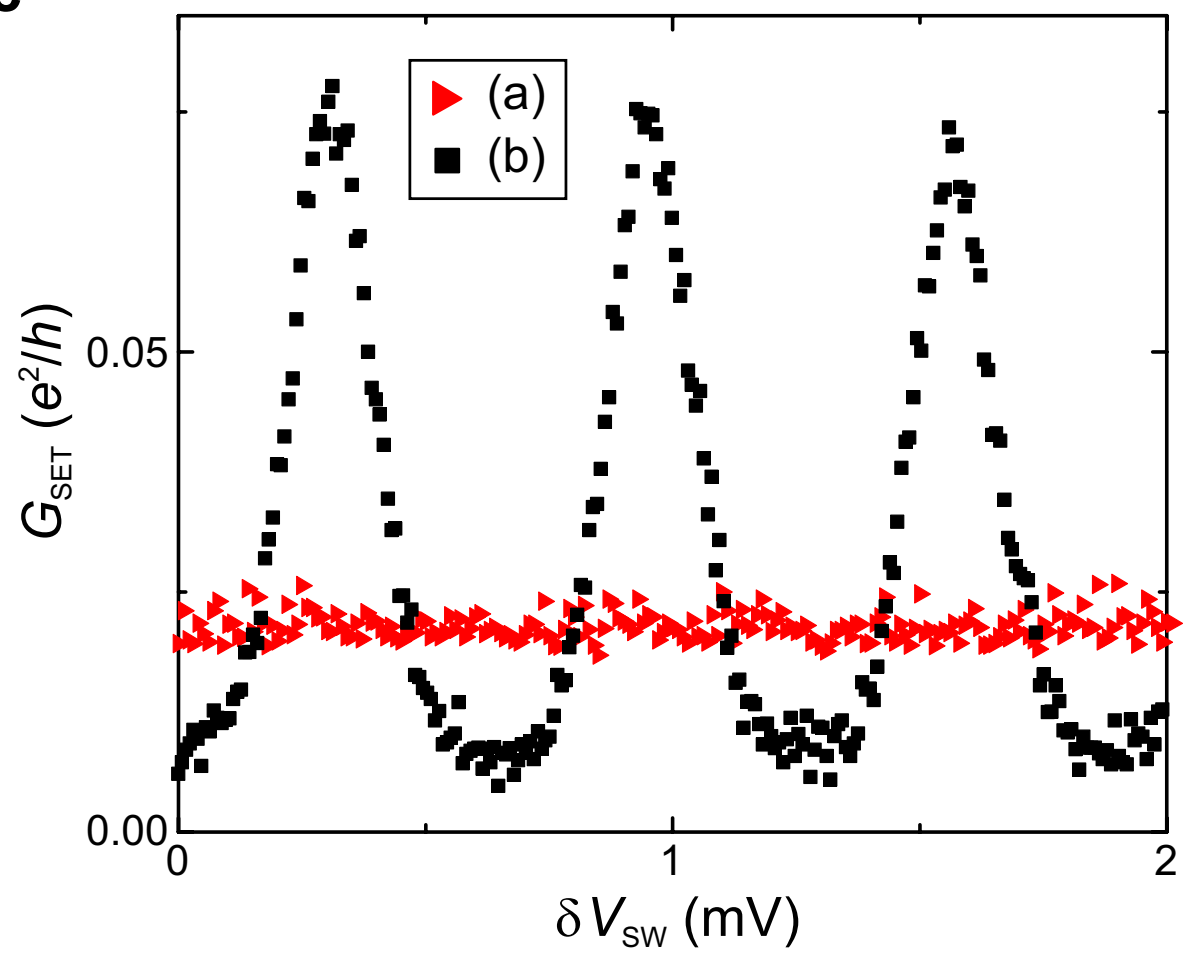

to the same intrinsic conductance $G_{\mathrm{R}}^{\mathrm{qpc}}=1.5 e^{2} / h$, which now decomposes into two non-ballistic channels of intrinsic transmission probabilities 0.7 and 0.8 . c, Sweeps of the device conductance are plotted versus gate voltage for the two configurations (a, red triangles; b, black squares).

Conductance oscillations are visible only in the configuration shown in $\mathbf{b}$, in the absence of a ballistic channel connected to the island. 